\title{
Thermo-hydrodynamic transport phenomena in partially wetting liquid plugs moving inside micro-channels
}

\author{
VYAS SRINIVASAN and SAMEER KHANDEKAR* \\ Department of Mechanical Engineering, Indian Institute of Technology Kanpur, Kanpur 208016, India \\ e-mail: samkhan@iitk.ac.in
}

MS received 7 July 2016; revised 14 October 2016; accepted 20 October 2016

\begin{abstract}
Single-phase as well as two-phase fluid flows inside mini/micro-channels and capillary tubes are of practical importance in many miniaturized engineering systems. While several issues related to single-phase transport are fairly well understood, two-phase systems still pose challenges for engineering design. The presence of gas-liquid interfaces, dominance of surface forces, moving contact lines, wettability, dynamic contact angle hysteresis and flow in confined geometries are some of the unique features of two-phase systems, which manifest into complex transport phenomena. While Taylor plug/bubble flow is a fairly common flow pattern in several micro-fluidic devices operating at low Bond number, the ensuing transport characteristics are complex and still not fully discerned. This review paper aims at highlighting the nuances and features of a unit cell of a Taylor plug flow, especially focusing on partially wetting systems, which are more common in engineering applications. Emphasis is given to a 'unit cell' flow system consisting of an isolated liquid Taylor plug with adjacent gas phase, confined in a capillary tube. Such a seemingly simple flow condition poses considerable challenges for discerning and modelling local thermo-hydrodynamic transport coefficients. Relevant background information and fundamentals are carefully scrutinized while summarizing the state-of-the-art. The role of wettability and dissipation near the contact line is highlighted via available experimental and simulation results. Local momentum and heat transfer exchange processes during the motion of an isolated plug of partially wetting liquid moving inside a capillary tube are delineated.
\end{abstract}

Keywords. Mini/micro-channel; capillary tubes; Taylor plug flow; unit cell; partial wetting; three-phase contact line; contact angle hysteresis; pressure drop; sensible heat transfer.

\section{Introduction}

Research in the area of two-phase flows in mini/microchannels has increased due to emerging applications in diverse branches ranging from bio-medical to thermal management of electronics and space systems, water management of fuel cells, micro-two-phase heat exchangers and reactors, nuclear rod bundles, DNA separation and analysis, digital microfluidics, lab-on-chips, micro-fluidic devices, loop heat pipes, etc. [1-6]. The enhanced transport properties and the compactness of these systems are highly desirable in the emerging trends of miniaturization and energy efficiency. Transport mechanisms of heat, momentum and mass under two-phase flow conditions in mini/ micro-geometries are greatly affected by the flow patterns that emerge in the system. These flow patterns are primarily decided by the interplay of surface, viscous, inertial and gravity forces. The scaling of these forces provides key insights into the underlying physics of two-phase flows. Studies have suggested that, for a liquid system to be

*For correspondence completely dominated by surface forces over gravity, it is required that the Bond number $(\mathrm{Bo})$ of the system lie below a value of approximately $2[7,8]$. The Bond number is thus defined as

$$
B o=\frac{\text { gravity force } / \text { area }}{\text { surface force } / \text { area }}=\frac{\left(\rho_{l}-\rho_{v}\right) g D}{\sigma / D}=\frac{\left(\rho_{l}-\rho_{v}\right) g D^{2}}{\sigma} .
$$

Thus, the Bond number of the system can decide whether the flow falls under the classification of mini/micro-channel. In two-phase systems with low Bond number, local interfacial characteristics will play a dominant role in the ensuing transport. The two-phase flow systems discussed in this review will all be in such mini/micro-regime, where $B o<2$ [9]. Bretherton [10] established the dominance of capillary forces in such systems, where the liquid-phase velocity was low. The transport phenomena in such system are governed by surface forces near the interface and the viscous forces in the bulk liquid. Hence, the scaling of surface forces to viscous forces dictates the shape of the 
interfaces or menisci. Capillary number $(\mathrm{Ca})$ is thus defined as

$$
C a=\frac{\text { viscous force } / \text { area }}{\text { surface force } / \text { area }}=\frac{\mu V / D}{\sigma / D}=\frac{\mu V}{\sigma} .
$$

Weber number signifies the dominance of inertial force of the fluid to its interfacial force (surface tension). Flows with high Weber and Capillary number have profound implications on the meniscus shape, hence the transport characteristics, and this is interlinked with the two-phase flow patterns that may eventually emerge. Weber number is defined as

$$
\text { We }=\frac{\text { inertia force } / \text { area }}{\text { surface force } / \text { area }}=\frac{\rho V^{2}}{\sigma / D}=\frac{\rho V^{2} D}{\sigma} .
$$

These three non-dimensional numbers dictate the majority of the hydrodynamic phenomena like the flow pattern, pressure drop, liquid thin film formation and drainage, etc., in two-phase systems in $\mathrm{mini} /$ microscale geometries. Some of the prominent possible two-phase flow patterns in mini/micro-channel are depicted in figure 1a. These flow patterns are controlled by the externally imposed gas-liquid superficial velocities, or governed by the applied heat flux, as in case of flow boiling [11].

In this review we focus our attention on the nuances of unit cells of Taylor liquid plugs flowing in confined geometries, i.e., capillary tubes or mini/micro-channels. The overall organization of the review is as follows: (i) section 2 will shed light on characteristic features of
Taylor plug flow; (ii) section 3 will discuss the importance of meniscus contact angles, spreading and wettability, moving contact lines (CLs), contact angle hysteresis $(\mathrm{CAH})$, thin-film formation and dewetting, which are of importance to model Taylor plug flows and (iii) section 4 will discuss the motion of Taylor plug systems under different conditions, highlighting the need for discerning local dynamics. Finally, we bring out the unresolved issues, future scope and direction in the art.

\section{Dry and wet regimes of Taylor plug flow}

One of the most prominent and interesting regimes of interfacial two-phase flow in a capillary tube is the Taylor flow or capillary slug flow, which is characterized by long gas/vapour bubble slugs separated by liquid plugs. Due to its inherent complexities and wide applications, this regime has been studied and reported extensively by many researchers, ever since the classical study by Fairbrother and Stubbs [12]. Taylor [13] reported an experiment where a viscous fluid was made to drain out of a capillary tube by a long gas bubble. The experiment reported that the entire liquid does not drain out but a film of liquid is always deposited on the tube wall. This work scaled the thickness of the drained film laid down as $\delta \propto(\mathrm{Ca})^{1 / 2}$. It is known from quite some time now that when a gas bubble displaces a wetting fluid that is filled in a capillary, a liquid film is deposited on the capillary between the gas bubble and the (a) Taylor bubble flow
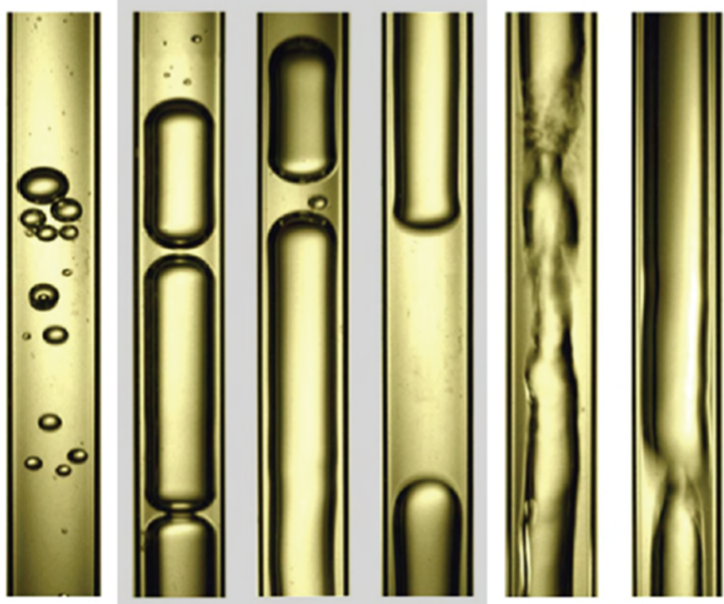

Increasing gas/vapour superficial velocity (b)
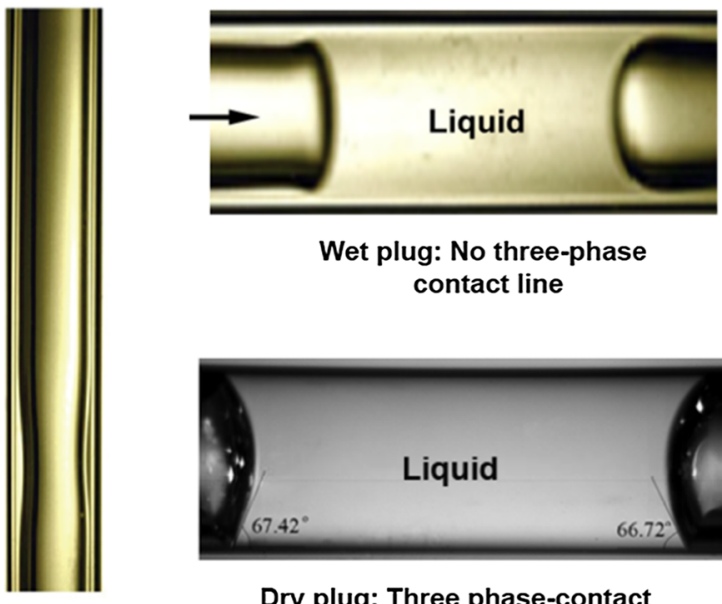

Wet plug: No three-phase contact line

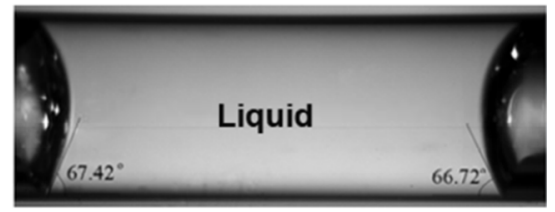

Dry plug: Three phase-contact line at liquid-gas-solid interface

Figure 1. (a) Different possible flow patterns in a capillary tube/micro-channel based on gas superficial velocity [9]. (b) Case of 'wet' Taylor-bubble flow, where a region of thin film exist around the gas bubble, eliminating the presence of contact line. (c) 'Dry' Taylor plug, observed when the solid is not pre-wetted by liquid and there is no thin-film around the gas slug, leading to the formation of threephase contact line [75]. 
inside wall $[14,15]$, as shown in figure $1 \mathrm{~b}$. Under certain circumstances (which may include wettability conditions between the solid wall and the liquid, shear flow, capillaryinduced instabilities, dewetting due to surface inhomogeneity, etc.), especially at low Capillary numbers, this liquid film surrounding the gas/vapour bubble may not get formed in a regular fashion, resulting in a flow with a dryplug regime, as shown in figure 1c. Hence, primarily two kinds of Taylor plug regimes appear in real-time engineering situations: wet and dry, respectively, with and without a liquid film, surrounding the adjoining gas/vapour bubbles (figure $1 \mathrm{~b}$ and $\mathrm{c}$ ). Usually the distinction between these two flow situations can be done on the basis of Capillary number; for $\mathrm{Ca}<10^{-3}$ (under flow boiling situations, liquid films surrounding the vapour bubbles can also dry out due to the input heat flux, resulting in 'dry' Taylor plug flows), dry plug regime is frequently encountered $[16,17]$. In the case of dry-plug motion, the respective menisci form distinct leading (advancing) and lagging (receding) contact angles, and the ensuing three-phase CL interactions lead to unique dissipation/transport rates of species (mass, momentum and energy) close to the menisci, as shown in figure $1 \mathrm{~b}$ and c. Numerical and experimental estimations of the pressure drop for the wet plug regime have been proposed [18-20]. However, hydrodynamics for the case of a dry plug regime has been scarcely studied, despite the fact that at low liquid flow rates, dry-out condition has been reported by various researchers [21-23]. In particular, such flows are frequently encountered during micro-fluidic operations involving gas/vapour-liquid interfaces.

This paper will deal with 'local' transport behaviour of a 'unit cell' of such dry Taylor plug flows. A unit cell is defined as a single active unit of Taylor flow, that is, one liquid plug, surrounded on both sides by gas bubbles (slugs). Several findings on the hydrodynamics and thermal transport of a unit cell are explained. The purpose is to shed light on the local behaviour of liquid plug in a mini-channel and to bridge the gap in understanding the physics of such flows.

\section{Contact line motion: scales and regimes}

Transport phenomena related to the 'dry' regime of the Taylor plug flow are strongly affected by the shape of the menisci defined by the apparent contact angle and the CL dynamics. Here, we present a short review of the relevant associated concepts.

\subsection{Static contact angle}

Instances of finite contact angles between liquid and solid phases occurring at the three-phase CLs are ubiquitous. Examples range from everyday experience of capillary rise of liquids in straw, raindrops sitting/sliding on leaves or automobile windscreens, dropwise condensation process, etc. In many of these systems, surface forces dominate. From a thermodynamic viewpoint, surface tension is the manifestation of the intermolecular forces defined at an interface separating the two phases, given by the Gibbs free energy per unit area [24]. The mechanical viewpoint of surface tension is in terms of the force required per unit length to create a fresh interfacial surface [25]. Ideally, the shape of a liquid droplet is determined by the resultant of the three respective surface tension forces in-between the three phases, respectively, acting on the CL. The equilibrium shape of liquids inside a capillary tube or a puddle of liquid on a solid surface is then decided by the balance of surface tension forces (which tries to minimize the surface area by resisting the need to spread) and gravity (which tries to spread). The scaling of this is given by the capillary length scale, as given by Eq. (2): $l_{c}=\sqrt{\sigma / \rho g}$, which is typically on the order of $10^{-3} \mathrm{~m}$ for most liquids. This means that a large drop will spread till its characteristics dimension corresponds to capillary length scale, where surface forces and gravitational forces get balanced (during the transience when spreading is taking place, the internal viscous forces also come into play). The resulting equilibrium static contact angle (the tangent from the threephase CL to the interface) of the liquid drop thus formed is given by Young's law as follows:

$$
\sigma_{l v} \cos \theta_{e q}=\sigma_{s v}-\sigma_{s l} .
$$

This is based on the force balance of solid-vapour, solidliquid and liquid-vapour surface tensions [26] at the CL. Thus, the liquid drop, depending on the surface energies involved, can take different shapes, as shown in figure 2a. An interesting case appears when the contact angle tends to zero, i.e., the liquid completely wets the solid surface due to the high surface energies of solid-liquid and solid-vapour compared to that of liquid-vapour interface. Most silicone oils show such 'lyophilic' behaviour on clean glass surfaces. Another asymptotic limit is when the contact angle tends to $180^{\circ}$, in which case the liquid-vapour surface energy is so high that it can overcome the solid-liquid and solid-vapour surface energies. This can be commonly seen in case of water droplets on lotus leaves (hydrophobic condition) or with oil droplets placed on Teflon-coated nonstick pans (lyophobic condition). The wettability of a liquid drop placed on a solid surface can tremendously vary based on the surface energy of the solid; a complete range from lyophilic to lyophobic behaviour can be achieved by suitably changing the surface energy distribution by physical and/or chemical texturing or treatment [27]. Such spreading behaviour can be described by defining a spreading coefficient $(S)$ as follows: $S=\sigma_{s v}-\left(\sigma_{s l}+\sigma_{l v}\right)$, which characterizes the interaction energies at the interface. If $S=0$, the liquid drop wets the surface completely, forming a thin film. However, when, the surface energies are moderate, which is common in many engineering applications, $S<1$ 

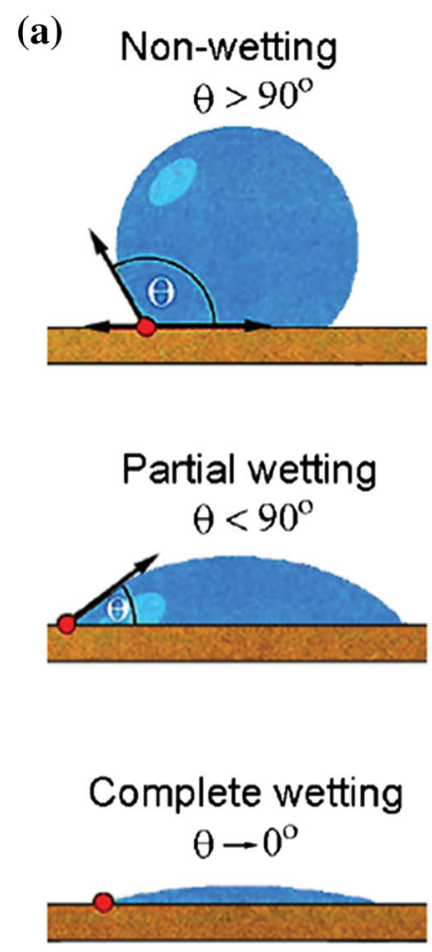

(b)

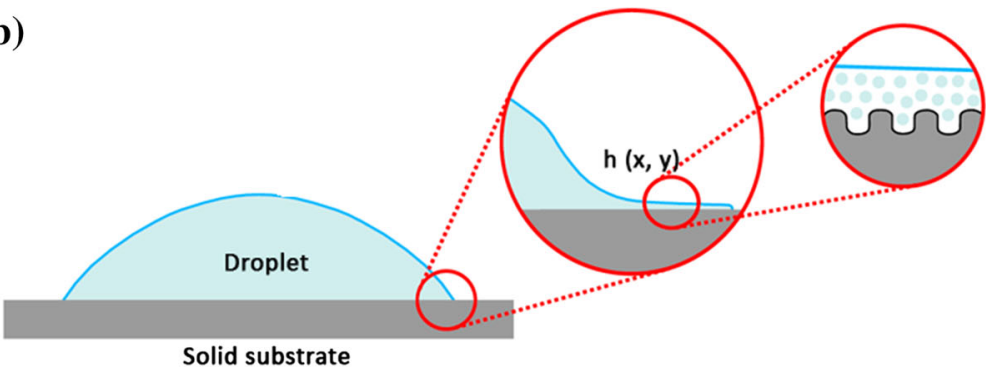

(c) Bulk drop region

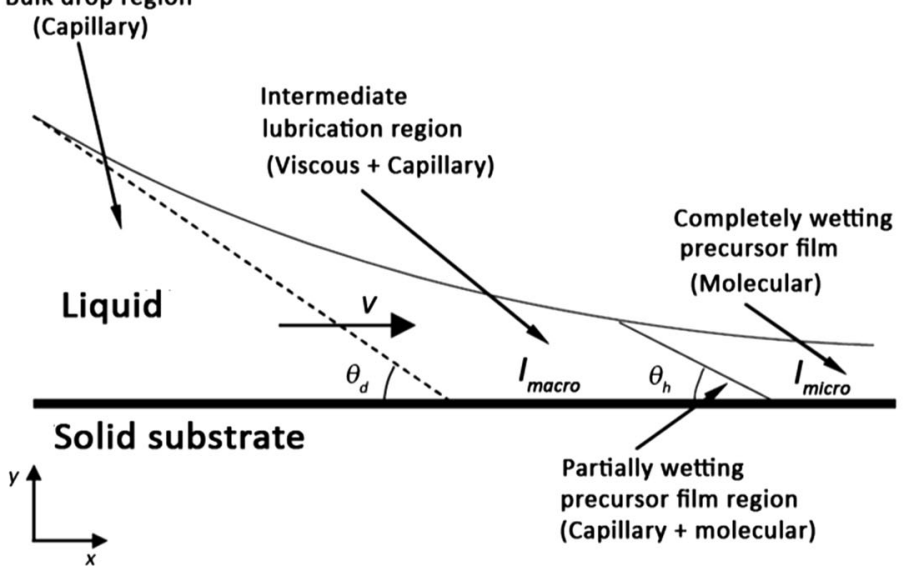

Figure 2. (a) Three different cases of wetting of drop on a solid substrate [9]. (b) Magnified view near the microscopic region of contact line. (c) The moving contact line for the case of complete and partial wetting, with each region showing the interaction of different forces involved.

and the liquid forms a definite contact angle, and is said to be in a condition of 'partial' wetting. The review by Bonn et al [27] provides valuable understanding about the wetting phenomena. It should be noted that the equilibrium static contact angle, as referred to here, is actually the 'apparent' or 'macroscopic' contact angle, since it is usually measured through high-resolution image of drops (or using a goniometer), where the CL is not clearly resolved. In fact, contact 'line' (CL) is conceptually an abstract concept, as there exists no real 'line'. While we assume a fluid continuum for analyses, in reality, thermally agitated discrete molecules are present at the interface and the threephase ' $C L$ ' region. Hence, the exact location of liquid contact on the solid substrate may vary on microscopic scales and the word 'line' to describe it is a misnomer [28]. The definition and usefulness of the apparent contact angle is dealt extensively by Decker et al [29].

The apparent contact angle may not always be the same on both sides of the drop (when the droplet is viewed on a $2 \mathrm{D}$ projection, as is usually done by measuring instruments such as goniometer cameras). For a general 3D droplet, the contact angle may vary in the azimuthal direction, as we move along the three-phase CL. Such behaviour is attributed to several reasons linked with chemical and physical inhomogeneity. The difference in the observed apparent contact angles of the static droplet is usually termed as static contact angle hysteresis (CAH).

In most engineering applications, such as two-phase flow in mini-channels, dropwise condensation on surfaces, liquid jet or droplet impingement, pool boiling, etc., interfaces as well as three-phase CLs are in motion. On many instances, the fluid-solid combination renders a state of partial wetting. The motion of such partially wetting fluid interfaces, which sounds seemingly simple, is a classical problem that questions the Navier-Stokes (NS) equation of continuum fluid mechanics. Under such situations, the concepts presented in this section need to be extended to include the effect of CL velocity on the interfacial shapes and resulting contact angles, a brief review of which is presented next.

\subsection{Moving contact lines: dynamic angles}

If the CL is moving relative to the solid, the resulting contact angle is called as apparent dynamic contact angle. Huh and Scriven [30] showed that the application of no-slip boundary condition at the moving CL leads to unrealistic results. If the no-slip boundary condition is applied at the contact point, then streamlines make a sharp turn to satisfy conservation of mass, resulting in a diverging velocity 
gradient when approaching $h=0$, as shown in figure $2 \mathrm{~b}$. This implies that the resulting shear stress at the contact point, which is scaled as $\tau_{w} \propto \partial V / \partial h$, diverges and a mathematical singularity arises, i.e., a liquid drop can never move over a solid substrate since it requires infinite force to do so. However, this argument negates our daily observations. Extensive review of the different mathematical models to remove the CL singularity is dealt by several authors, with the prime focus on predicting the interface curvature due to strong viscous bending in the vicinity of moving CL (for example, see [31-33]).

Further, the studies of Rose and Heins [34], Dussan and Davis [35] and Tanner [36] revealed that the apparent dynamic contact angle of a spreading/moving droplet on a solid substrate depends on the CL speed (i.e., Capillary number) itself. Similarly, even for moving interfaces in capillary tubes/confined geometries, several classical studies (for example, see $[37,38]$ ) have indicated the dependence of apparent contact angle on the Capillary number.

In many systems, static interfaces move and eventually attain finite velocities. Classical theories make a distinction between two regimes, i.e., low Capillary number systems (typically $\mathrm{Ca}<10^{-3}$ ) wherein the transport is primarily guided by viscous and surface tension forces, and high Capillary number systems, wherein inertia forces also start playing a role. For the latter cases, the Weber number, as defined by Eq. (3), also needs to be considered. When interfaces get transformed from a static condition to a dynamic condition, the resulting contact angles also get affected. As depicted in figure 3, one can see that this change in the contact angle consists of two regimes. First, there occurs a change in the contact angle when the interface tends to move in a given direction due to the application of an external force. While the force deforms the interface, the CL may still remain at the same location. This changes the 'advancing' contact angle (the contact angle made by the portion of the interface at the advancing CL, which will experience a dry solid surface if motion is initiated) as well as the receding contact angle (the contact angle made by the portion of the interface at the receding CL, which will experience a wet surface if motion is initiated). Hence, there occurs a net difference in the advancing and receding contact angles, on application of an external force, even before any movement of the CLs has taken place, which is called as the static CAH. Such a phenomenon is routinely observed, especially in partially wetting fluids on surfaces that are physically or chemically inhomogeneous. It can be interpreted as the difference between the maximum (advancing) and minimum (receding) contact angles for which a (local) minimum exists in the Gibbs free energy [39]. These metastable angles are formed due to the high energy barriers present at the surface, which prohibit the drop to minimum energy conditions. This static hysteresis is generally highly stable, i.e., a droplet can be deposited in a state where it maintains a contact angle between the two limiting angles and resists the motion (shape of drops on inclined plane, without motion) [40]. Once the interface attains a finite velocity, the hysteresis may continue, which will now be termed as 'dynamic contact angle hysteresis (DCAH)'. It now depends on the Capillary number, which affects the local interface shapes. We will revisit this DCAH phenomenon in section 3.3 while discussing interface dynamics. In the next section, the hydrodynamic approach of resolving the singularity and modelling of hysteresis is briefed, owing to its wide applicability in engineering systems [41, 42].

\subsection{Hydrodynamic model}

The hydrodynamic model overcomes the CL singularity elegantly in the framework of continuum fluid mechanics. This is achieved based on the fact that, for a completely wetting drop, a 'precursor' film always exists ahead of the moving CL, allowing for a small 'slip' length near the wall $[43,44]$, as depicted in figure $2 b$. Referring to figure $2 \mathrm{c}$, for the purpose of analysis, the region close to the $\mathrm{CL}$ is usually divided into three zones, viz., the microscopic region, consisting of the precursor layer dominated by molecular level force fields (typically of 3-100 nm), the mesoscopic zone, where the bulk liquid viscosity starts appearing, and finally, the macroscopic zone, where the transport is affected by capillary and viscous forces and regular NS equations can be routinely applied. Based on the hypothesis of such precursor films (typically ranging from 3 to $100 \mathrm{~nm}$ ), the long range van der Walls forces will play an active role in deciding the shape of the local interface and flow immediate to the CL [35, 45, 46]. In short ranges of the precursor film $\left(3-100 \mathrm{~nm}, l_{\text {micro }}\right.$ in figure 2c), the thickness of the film is governed by the disjoining pressure [47]. The disjoining pressure in the precursor film arises from the molecular attractive force between the liquid thin film and the solid substrate. On this nanometric scale, the attractive forces between the liquid layer and the solid substrate dominate over the net cohesive force of liquid, and hence, a stable film is favoured, which leads to a pressure difference between the film formed and the bulk phase. The measure of this difference in pressure is the disjoining pressure $(\Pi)$, given by Israelachvili [48]:

$$
\Pi=f(A, h)=\frac{A}{6 \pi h^{3}}
$$

where $A$ is the Hamaker constant, typically of the order $10^{-19} \mathrm{~J}$, which characterizes the van der Waal's interaction energy between two surfaces. Eq. (5) can therefore be used to predict the thickness of the precursor film $(h)$, which arises primarily due to the intermolecular forces between the liquid and solid [49, 50]. This framework provides the intermolecular origins for a finite contact angle. Above approximately $100 \mathrm{~nm}$, the effects of long range van der Walls forces weaken and the interface curvature is 
(a) Hysteresis in drop motion

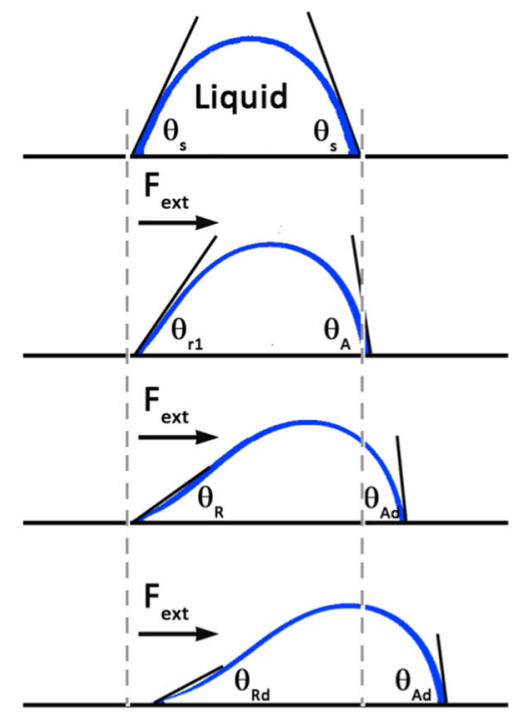

(b) Hysteresis in liquid plug motion

\section{Qualitative description}

No external force

$\mathrm{V}_{\mathrm{CL}}=0$

$\theta_{\mathrm{R}}<\theta_{\mathrm{s}}<\theta_{\mathrm{A}}$

$\mathrm{F}_{\text {ext }}=$ Constant

$\mathrm{V}_{\mathrm{CL}}=\mathbf{0}$

$\theta_{\mathrm{R}}<\theta_{\mathrm{r} 1} ; \theta_{\mathrm{A}}$ attained

$\mathrm{F}_{\text {ext }}=$ Constant

$\mathrm{V}_{\mathrm{CL}}=\mathbf{0}$

$\theta_{\mathrm{R}}$ attained; $\theta_{\mathrm{Ad}}>\theta_{\mathrm{A}}$

$\mathrm{F}_{\mathrm{et}}=$ Constant

$\mathrm{V}_{\mathrm{CL}}^{\text {ext }}>0$

$\theta_{\mathrm{Rd}}<\theta_{\mathrm{R}} ; \theta_{\mathrm{Ad}}>\theta_{\mathrm{A}}$

(c) Schematic of static and dynamic hystereis
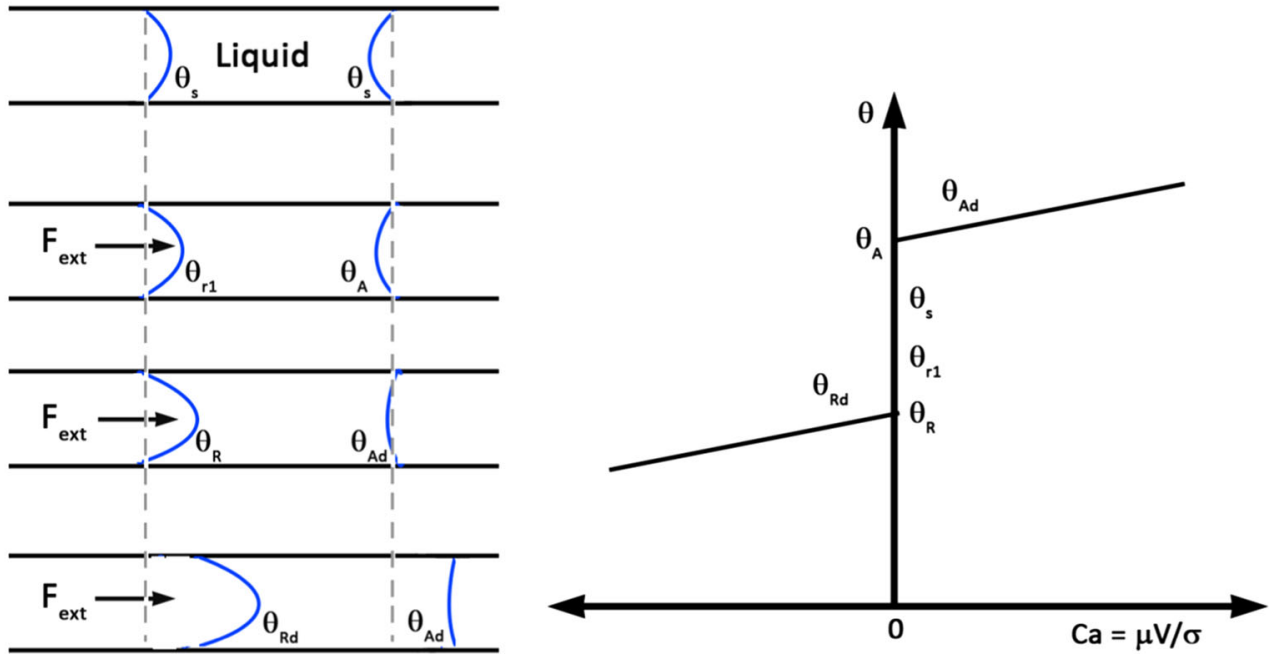

Figure 3. (a) A drop starting to move under the influence of external force, where interface is seen to deform even before bulk motion of drop. The complete motion of drop begins after both the meniscus has deformed to the limit of static contact angle hysteresis given by $\theta_{A}$ and $\theta_{R}$. (b) Hysteresis in liquid plug motion inside a capillary tube, which exhibits similar qualitative behaviour as drops. (c) Variation of contact angle hysteresis with Capillary number, where the deformation of static meniscus at $\mathrm{Ca}=0$ is the static contact angle hysteresis, the various scenario in case $\mathbf{a}$ and $\mathbf{b}$ are qualitatively represented.

controlled by the dissipation due to viscous and capillary forces [51, 52]. In this mesoscopic region, the flow is modelled by the lubrication approximation of the NS equation, which was rigorously developed by Hoffman [37], Voinov [53] and Cox [54], near the vicinity of moving $\mathrm{CL}$. The emerging equation describes the relation of apparent dynamic contact angle as a function of CL velocity (Capillary number), which is now commonly called the Cox-Hoffman-Voinov model, given by (see figure 2c)

$$
\theta_{d}^{3} \propto 9 C a\left[\ln \left(\frac{l_{\text {macro }}}{l_{\text {micro }}}\right)\right] .
$$

This relation relies on matching of interface curvature based on balance between viscous and capillary forces at different length scales, viz. the adsorbed layer, the thin precursor film region and the bulk liquid interface. Thus, it requires a cut-off length that demarcates the influence of intermolecular effects $\left(l_{\text {micro }}\right)$ and capillary effects $\left(l_{\text {macro }}\right)$. It has to be noted that this equation is not universal and is 
valid for completely wetting systems, for which a precursor film exists.

For partial wetting liquids, the existence of the precursor film is itself debated $[45,55]$. However, the equation holds well for predicting apparent dynamic angles for liquids with apparent static contact angles up to about $30^{\circ}$ [46]. The Cox-Voinov relation can predict both advancing and receding apparent angles, as follows:

$$
\begin{aligned}
& \theta_{A}=\theta_{s}+9 C a \ln \left(\frac{\beta l_{\text {macro }}}{l_{\text {micro }}}\right) \\
& \theta_{R}=\theta_{s}-9 C a \ln \left(\frac{\beta l_{\text {macro }}}{l_{\text {micro }}}\right)
\end{aligned}
$$

where $l_{\text {macro }}$ is typically the macroscopic length scale corresponding to the capillary length scale $\left(l_{\text {macro }}=\sqrt{\sigma / \rho g}\right)$ and $l_{\text {micro }}$ corresponds to the microscopic length scale (typically on the order of $10 \AA$ ); $\beta$ is a non-universal constant typically in the range of $1-10$ (for example, see $[32,33,56])$. The difference between the dynamic advancing angle and the receding angle leads to dynamic hysteresis, as explained earlier. This can be seen when a solid plate is slowly moved inside a bath of stagnant liquid (forming an advancing angle) and pulled back from the bath at the same velocity (forming receding interface). Figure $3 \mathrm{a}$ and $\mathrm{b}$ shows CAH, taking into account both its static and dynamic parts, for the case of droplet as well as a liquid plug confined inside a tube. It has to be noted that unlike static $\mathrm{CAH}$, which is due to the surface morphology, the dynamic $\mathrm{CAH}$ is additionally due to the motion of $\mathrm{CL}$ itself. In this scenario, the receding dynamic angle moves over a pre-wetted region of solid substrate, where the advancing front has already moved. As seen from figure $3 c$, a liquid drop/plug may take any static contact angle in the range of its static hysteresis $\left(\theta_{R}\right.$ and $\left.\theta_{A}\right)$. Upon influence of an external force, the droplet/liquid plug first deforms with no bulk motion at CL till the static hysteresis is overcome. Once this stage is surpassed, the CL moves and the dynamic hysteresis can be explained in terms of Eqs. (6) and (7) $[57,58]$. As a consequence of this hysteresis, the curvatures at the advancing and receding interfaces are different, which leads to an additional capillary pressure, which is modelled as [59] follows:

$$
\Delta P_{\text {cap }}=\frac{2 \sigma}{r}\left(\cos \theta_{R}-\cos \theta_{A}\right) \text {. }
$$

Another practical artefact of CL moving over the real surface is the effect of pinning, where the contact angle pins on the local surface heterogeneity, which is stronger than the force that causes the motion, as depicted in figure 4. This makes the interface to deform locally and then jump back to motion, when the local resistance is overcome. Modelling of such pinning effects is done based on critical wetting/dewetting phenomena, where the jump is modelled as a second-order phase transition [60, 61]. (a)

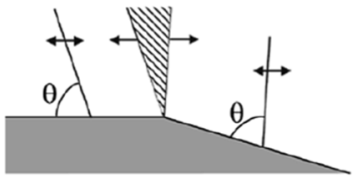

(b) Pinned interface

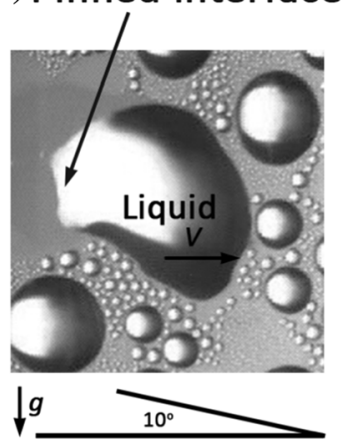

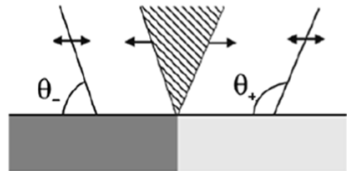

(c) Pinned interface

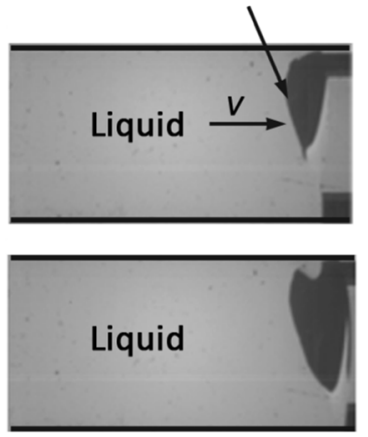

Figure 4. (a) Pinning of contact line of a strong physical or chemical discontinuities in a surface [9]. (b) Pinned interface of a moving droplet during dropwise condensation over a solid surface, which is inclined at $10^{\circ}$ to the horizontal. (c) Pinned interface of a liquid plug moving inside a capillary tube.

For systems exhibiting complete wetting, it is now clear that when a liquid drop moves, there is going to be a precursor film. Also, when a stationary plug of fully wetting liquid kept inside a capillary tube is made to move by application of an external force, then a uniform thin film gets drained at the receding interface [10, 13, 62], as shown in figure $5 \mathrm{a}$. This effect is used in practical applications of dip coating of plates and capillary tubes. The thickness of such films is given by Taylor [13], and later modified by Aussillous and Quéré [62]:

$$
\frac{h}{r}=\frac{1.34 C a^{2 / 3}}{1+1.34 * 2.5 C a^{2 / 3}} .
$$

Han and Shikazono [63] have extensively studied this thin-film thickness experimentally and their implication on hydrodynamics and heat transfer is well understood for Taylor plug flows through the work of Kreutzer et al [18], Morris [41] and Thome et al [11]. The additional pressure drop due to such a thin film is scaled by Bretherton [10] as follows:

$$
\Delta P=\frac{\sigma}{r}\left(1+3.88 C a^{2 / 3}\right) .
$$

However, for partially wetting liquids, such films may not be stable, leading to dewetting of the film (see figure $5 b$ ). This is due to the fact that for partial wetting liquids, which are forced to move over a solid surface, it is energetically favourable not to wet the surface, especially at higher Capillary number [64]. This dewetting phenomenon occurs due to spontaneous rupture of the thin film when its thickness is smaller than a critical 
thickness [65, 66]. Thus the thin film will undergo dewetting transitions by forming puddles of liquid mass, which has been extensively studied by Flitton and King [67] and Snoeijer and Eggers [68]; the condition for this dewetting to occur is given as follows:

$$
h_{c}=2\left(\sqrt{\frac{\sigma}{g \Delta \rho}}\right) \sin \left(\frac{\theta_{s}}{2}\right)
$$

where $h_{c}$ is the critical thickness of the film, below which instability causes the film to dewet and form small droplets/ puddles. This process of film formation and its subsequent dewetting eventually leads to instances of additional CL formation, requiring additional surface energy to be supplied. Hence, the pressure drop of a completely wetting liquid plug will be usually lower than that of a partially wetting liquid plug [69, 70].

Having understood the basis of contact angle and its dynamics, for completely wetting and partial wetting liquids, it is now clear that the forced motion of partial wetting liquids poses additional problems to be tackled in terms of its modelling (see the review by Snoeijer and Andreotti [56]). For example, in the absence of precursor film, how to describe the DCAH? This question is important in connection with the estimation of pressure drop required to move isolated liquid plugs in dry capillary tubes-such conditions are frequently encountered in many engineering systems, including lab-on-chip devices, biological flows, flows in fuel cell systems, pulsating heat pipes, near dry-out conditions during flow boiling, etc. (for example, see Khandekar et al [9], Rao et al [71], Safavieh and Juncker [72]). In this background of challenges in understanding the moving CLs for partial wetting liquids, the next section will discuss some of the experimental and simulation studies of such liquid plugs with regard to the pressure drop and heat transfer characteristics.

\section{Transport phenomena in unit cells: some representative results}

In this section, local transport phenomena associated with two different types of confined liquid flows having gas interface(s), with no phase change, will be discussed. The compiled results are primarily emanate from the laboratory of the authors, obtained in the recent past. The two different flow conditions addressed here are (i) single confined meniscus (one liquid-gas interface) initially at rest and then made to move inside a capillary tube by the application of an external force and (ii) an isolated liquid plug (having two liquid-gas interfaces), initially at rest, and subsequently made to move inside a capillary tube by the application of an external force. These two flow situations are described in figure 6.

\subsection{Hydrodynamics of a single meniscus moving in a confined channel}

To establish and understand the contribution of interfacial forces towards the transport behaviour of a confined isolated liquid meniscus, a micro-PIV investigation was carried out in a dry capillary tube of square cross-section $(500 \mu \mathrm{m} \times 500 \mu \mathrm{m})$ at low Capillary numbers (order of $10^{-06}$ ). A single meniscus of liquid-gas interface (waterair) was examined under micro-PIV as shown in figure 6a. The study revealed quantitative information on the local flow characteristics and gave insight on the velocity field behind the meniscus. Figure 7 shows the flow field visualization of moving interface. The micro-PIV visualization shows that the flow near the meniscus becomes three-dimensional with both $v$ and $w$ velocities showing a double vortex, strength of the former being higher than the latter. This three dimensionality in the flow is due to the drag

(a)

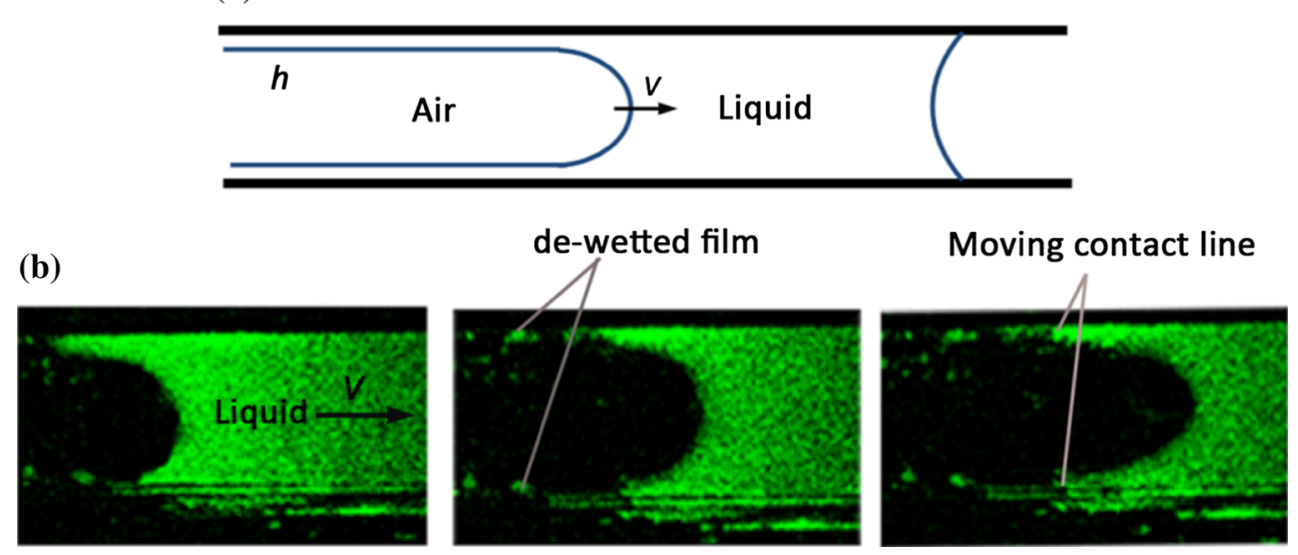

Figure 5. (a) Schematic of liquid film drained behind the receding interface of a completely wetting drop inside capillary tube. (b) Dewetted thin film during motion of receding interface of partial wetting liquid inside a capillary tube [75]. 
(a)

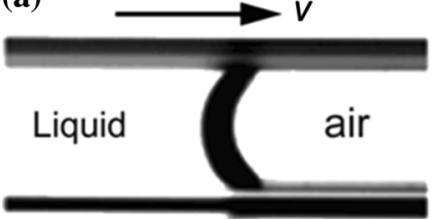

(b)

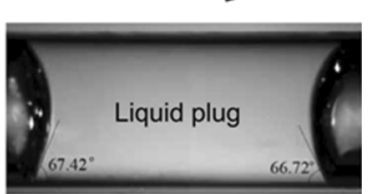

Figure 6. (a) Motion of a single confined interface of partially wetting liquid inside a capillary tube [74]. (b) Motion of partially wetting isolated liquid plug (with two distinct menisci) inside a dry capillary tube.

force on the contact surface with the side walls of the micro-channel and the fact that the meniscus must simultaneously obey the free surface boundary condition.

From the velocity profile (figure 7), it is clear that the bulk of liquid (far away from meniscus) follows a parabolic profile (Poiseuille-type flow), while as the meniscus is approached, it gets drastically altered (becomes flatter). The study thus suggests that only the Capillary number is not sufficient to predict the friction factor. Also, the meniscus profile (depending on the wettability) needs to be included for a more precise prediction of friction factor. Figure 8 demonstrates this by showing the variation of Poiseuille number $\left(C_{f} R e\right)$ along the wall, due to the steady motion of meniscus, for different wettabilities at the same Capillary number. This study reveals that the momentum flux coefficient increases rapidly as the three-phase CL is approached. Also, higher the wettability of the liquid on the tube material (characterized by the static apparent contact

\section{(a) u-velocity profile}
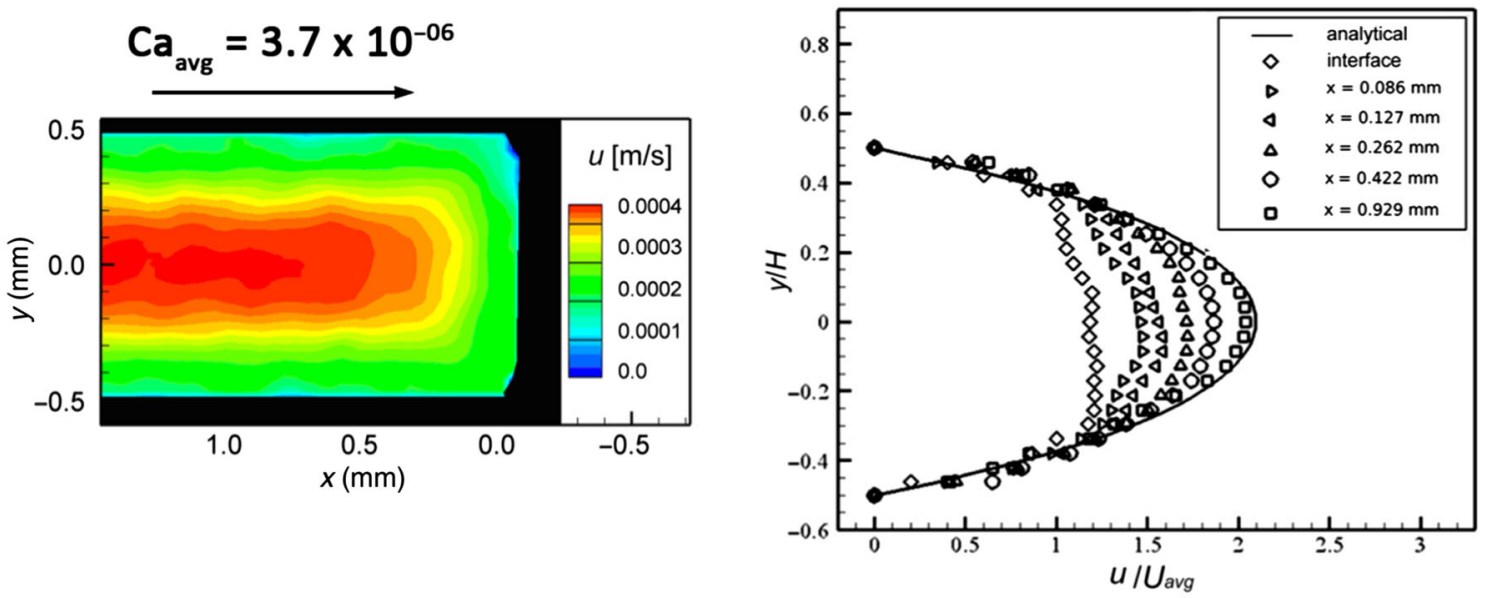

(b) v-velocity profile
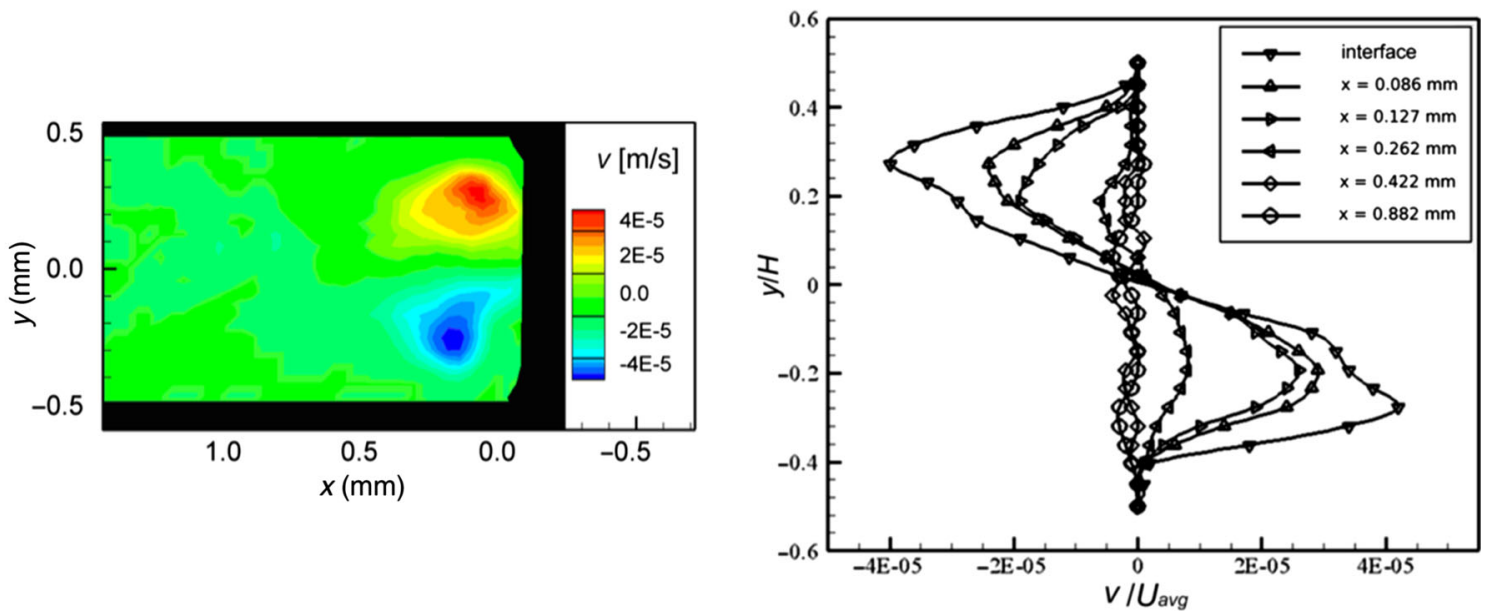

Figure 7. Micro-PIV result of (a) $u$-velocity field behind a single meniscus moving at a steady velocity $\left(\mathrm{Ca}=3.7 \times 10^{-06}\right)$ inside a capillary tube, with corresponding velocity profile at different location away from the interface. It can be seen that the $u$ velocity is disturbed near the interface and is not parabolic, (b) corresponding $v$-velocity profile, which shows a strong vortex just behind the interface, the $v$ velocity becomes zero far away from the interface. All the measurements have been performed at the mid-plane of the channel cross-section. $x$ is the stream-wise axial distance from the interface ( $x=0$ corresponds to the interface). Rana et al [74]. 
(a)

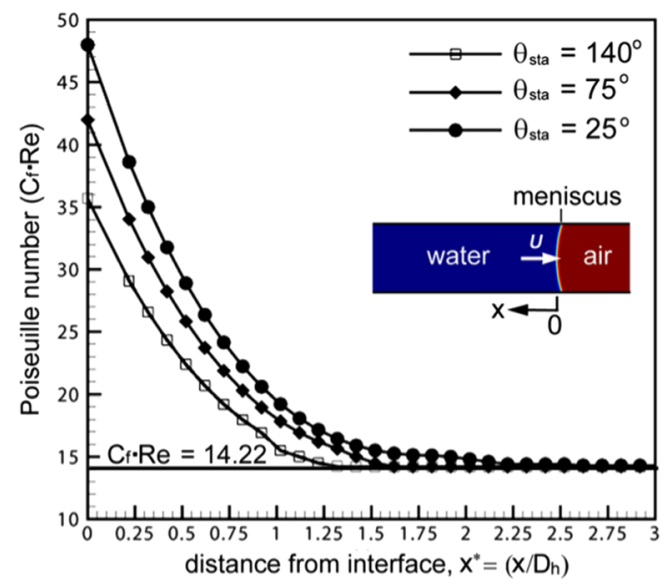

(b)

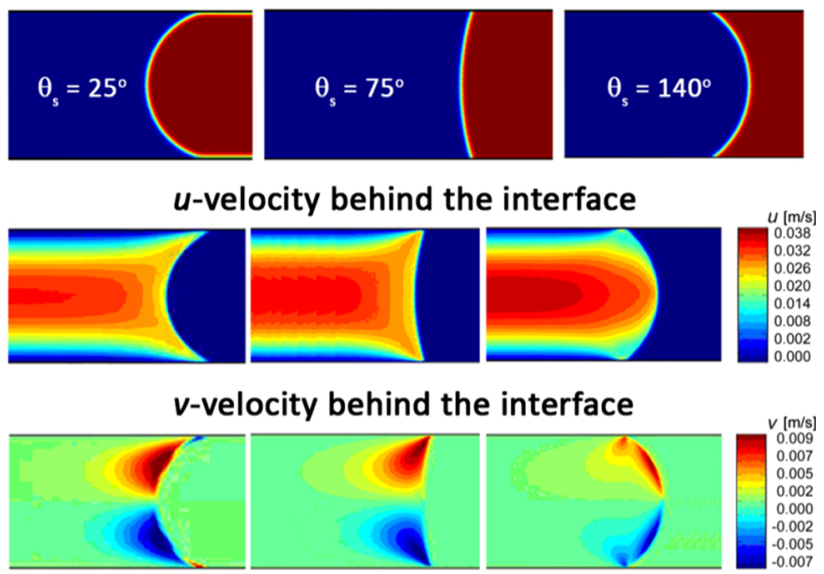

Figure 8. (a) Variation of Poiseuille number $\left(\mathrm{C}_{\mathrm{f}} \mathrm{Re}\right)$ experienced along the wall due to the steady motion of the meniscus, for the three cases of wettability respectively. (b) Simulation result of $u$-velocity and $v$-velocity profile behind the interface for three different wetting cases, it can be seen from the velocity field that high wetting case $\left(\theta_{s}=25^{\circ}\right)$ has a high disturbance in flow field behind the meniscus, hence showing higher Poiseuille number than low wetting case $\left(\theta_{s}=140^{\circ}\right)$. Rana et al [74].

angle), higher the value of the hydrodynamic friction factor close to the meniscus. The unique transport effects due to the presence of the meniscus are thus dependent on the static and dynamic wettability (and hence its local curvature/shape). The meniscus disturbs the otherwise existent Poiseuille flow in the liquid plug and this disturbance is confined to a distance of about $2 D_{h}-3 D_{h}$ away from the meniscus.

The dynamic shapes of isolated moving menisci between liquid-air systems inside a square mini-channel have been studied for water, ethanol, glycerine and silicone oil, with varying $\mathrm{Ca}$. It is shown that a drastic change in the dynamic shape of the meniscus can occur due to the imposed bulk velocity. Subsequently, using micro-PIV, liquid velocity distributions near the moving water-air meniscus were measured at the mid-plane of the channel, at low Capillary numbers. Near the liquid-air interface, the non-dimensional $u$-velocity profiles are flatter in nature, whereas on moving away from the meniscus the profiles follow the fully developed flow profiles, as in the case of conventional single-phase flow. The $v$ velocity is moderately dominant only close to the interface, although its absolute value is rather small as compared with the $u$ velocity. Formation of opposite vortices is clearly indicated by the $v$-profiles. Checking the continuity close to the interface indicated that the flow is indeed three-dimensional in nature in the proximity of the interface. It is highlighted that nearmeniscus local transport phenomena are critical in understanding the transport behaviour of microscale multi-phase systems. The experimental data for the flow field close to moving liquid-air interface are compared to those of numerical simulations. After benchmarking, the spreading coefficient is varied in the simulations to highlight its isolated effect on local flow transport characteristics. Close to the meniscus the flow clearly becomes three-dimensional with both $v$ and $w$ velocities showing a double vortex, the strength of the latter being an order-of-magnitude lower than the former. This leads to enhanced local transport. The simulations also reveal that controlling the wettability of the liquid can be an effective tool to control the viscous shear; the meniscus region of wetting liquids manifests stronger shear stress as compared with a non-wetting liquid moving at the same Capillary number (figure 8b). It is expected that the heat and mass transfer near the meniscus region will also be qualitatively affected in a similar fashion as that of the momentum flux transfer, taking the cue from Prandtl and Schmidt number analogy. Thus, it is concluded from this study that wettability of the fluid is an important parameter for species transfer under dynamic conditions near the meniscus regions in microscale geometries [73, 74].

Extrapolating the ideas from this study, to the case where a liquid plug moves in a capillary tube (with two distinct menisci as against only one meniscus), one may expect a highly wetting liquid plug to exhibit a higher pressure drop compared with a partially wetting liquid plug. The next section will scrutinize this expectation via a systematic study, delineating the effect of wettability and hysteresis on the local transport behaviour of confined liquid plugs.

\subsection{Thermo-hydrodynamics of liquid plug flow}

4.2a Hydrodynamics of isolated liquid plug in a minichannel: A liquid plug at rest, in equilibrium with its surrounding, forms a 'static' contact angle, which is the same 
(a)

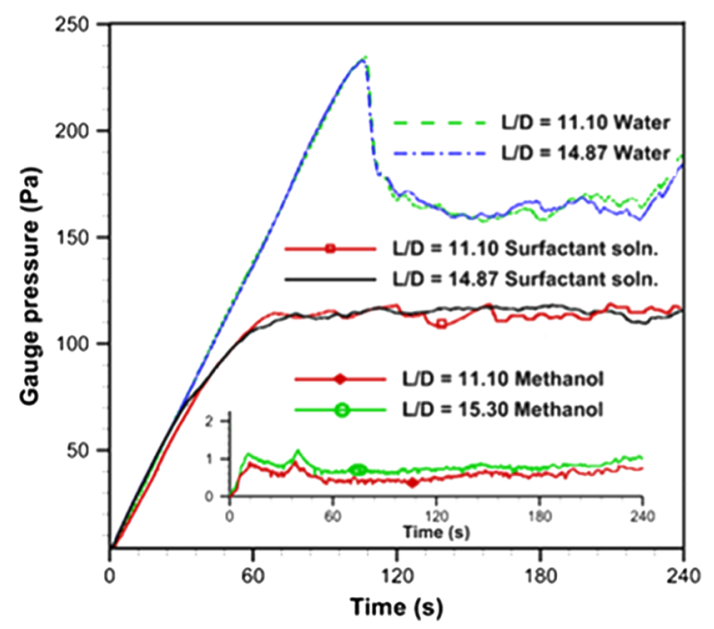

(b)

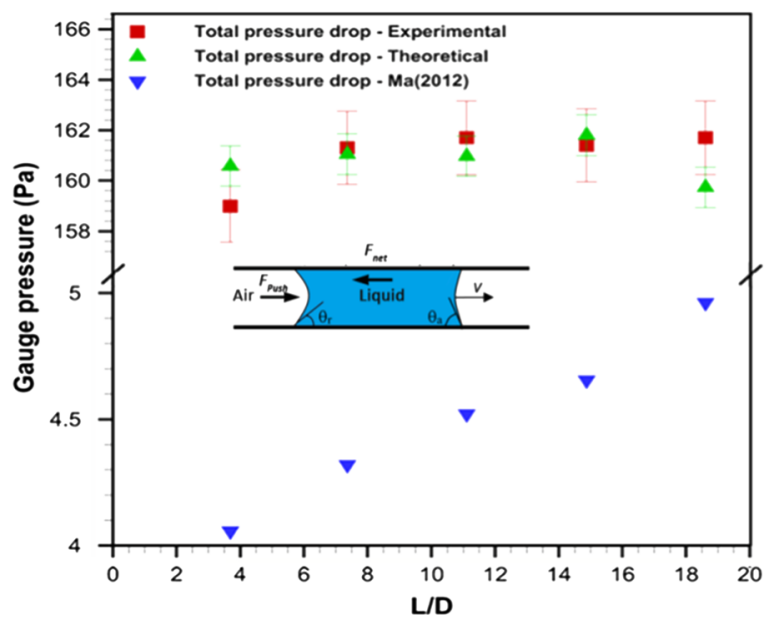

Figure 9. (a) Pressure drop required to push the liquid plug from rest till the attainment of steady state motion of three different fluids, as adapted from Srinivasan et al [75]. It is seen that water (having highest static contact angle compared to the three fluid used), has the highest steady state pressure drop. (b) Comparison of steady state pressure drop for water, predicted based on model proposed by Ma [79] and using the capillary pressure based on experimentally observed advancing and receding angles [Eq. (12)]. It is seen that the prediction of Eq. (12), closely matches the experimental pressure drop, whereas Ma's model is an order of magnitude away from it. (Inset) schematic of the liquid plug moving at steady terminal velocity.

on either side (since the liquid plug is in equilibrium with atmosphere on both ends and the system is isotropic). The menisci thus formed tend to hold the bulk liquid together against motion. To initiate motion of the liquid plug, it is pushed from one side, while the other end is kept open to atmosphere, creating pressure difference along the liquid plug. Due to the dominance of surface forces, the plug manages to stay at rest, despite the increasing pressure with time (analogous to 'static friction' in terms of solid motion). During this period, the force of pushing is resisted by the surface forces, which leads to deformation of menisci in response. An experimental study was carried out by Srinivasan et al [75] to understand the physics of motion of an isolated, dry liquid plug. The liquid plug was forced to move from one side by injecting air from a large reservoir at constant flow rate. Since the injection of air (gas bubble) was so low, the associated Capillary number was of the order $10^{-05}\left(<10^{-03}\right)$. Hence, a dry plug regime was typically maintained.

If the moving liquid plug inside the tube is infinitely long, the system can be easily modelled by conventional fluid mechanics of internal flows (incompressible fluid flow without any surface effects), giving a constant Poiseuille number, $\mathrm{Po}=C_{f} R e=64$, under laminar flow conditions. However, for liquid plugs of finite lengths, interfaces appear, and hence surface forces become important, especially at small Bond numbers $(B o<2)$. Then, the validity of classical Hagen-Poiseuille formulation is questionable and needs to be ascertained, in view of the local change in flow patterns in the close proximity of the interfaces, as has been highlighted in the earlier section and the add-on effect of surfaces forces acting at the interfaces. For example, it is known that the steady-state velocity of liquid plugs moving in a vertical capillary tube, driven by gravity, does not follow the Hagen-Poiseuille relation [76-78]. Bico and Quéré [76] have pointed out the importance of including the high viscous dissipation near the contact regions, which slows down the velocity of the liquid plug. The work of Srinivasan et al [75] involving motion of partially wetting liquid plugs in dry capillary tubes also reported strong deviations from the classical predictions of frictional pressure drop, which include only velocity-gradient-based hydrodynamic viscous friction. This study highlighted the significant contribution of capillary forces, in the overall budget of dissipative forces encountered during plug motion. For example, for the experimental velocity reported (the liquid being water), the steady state Poiseuille pressure drop was found to be $\sim 5 \mathrm{~Pa}$; however, the experimentally observed pressure drop reported was around $\sim 166 \mathrm{~Pa}$. Another interesting observation was that this pressure drop was independent of the $L / D$ ratio of the liquid plug, thus confirming the insignificant proportion of Poiseuille friction as compared with strong dissipation due to surface forces at, and in close proximity to, the interface. Figure 9 shows some of the important findings of the study.

A significant observation from figure $9 \mathrm{a}$ is that, independent of the $L / D$ ratio of the system, the deformations of the advancing and receding menisci remain identical, at steady-state motion of the liquid plug. Another interesting observation is the fact that highly wetting liquids show the 
least deformation of menisci, and hence require very low pressures drop for their motion (close to Poiseuille prediction), compared with partially wetting fluid such as water. This observation is in contrast with the understanding of single-meniscus study reported earlier, which considered only hydrodynamically originated friction and ignored the surface and interfacial effects. However, this can be explained by to the high capillary pressure difference involved due to $\mathrm{CAH}$ for non-wetting liquids, and the fact that there is a circulation pattern inside the liquid plug due to confinement of interface, which leads to additional pressure drop. The findings also suggest that for liquid with high apparent static contact angle, the hysteresis between the front (advancing) and back (receding) angle is high.

In a recent mathematical model for predicting pressure drop across a dry unit cell of Taylor flow, Ma [79] makes an attempt to appreciate the relative importance of the two major sources of pressure drop during liquid plug motion. In brief, the model equations are as follows (for details, please refer to the original source [79]):

$$
\Delta p_{\text {drive }}=p_{1}-p_{2}=\frac{32 \mu L V}{D^{2}}+\frac{4 \sigma\left(\cos \theta_{R}-\cos \theta_{A}\right)}{D}
$$

where the first term represents the frictional component due to wall shear assuming a fully developed Hagen-Poiseuille flow approximation in the liquid plug. The second term represents the additional pressure drop due to capillary forces. During further development of the model, Ma [79] invokes the functional relationship of the advancing contact angle with the static contact angle and the imposed meniscus velocity (i.e., $\mathrm{Ca}$ ), given by Bracke et al [80] as follows:

$$
\cos \theta_{A}=\cos \theta_{s}-2\left(1+\cos \theta_{s}\right) C a^{0.5}
$$

to reformulate Eq. (12) as follows:

$$
\begin{aligned}
\Delta p_{\text {drive }}= & \left(p_{1}-p_{2}\right)=\frac{32 \mu L V}{D^{2}} \\
& +\frac{4 \sigma\left(\cos \theta_{R}-\cos \theta_{s}+2\left(1+\cos \theta_{s}\right) C a^{0.5}\right)}{D}
\end{aligned}
$$

or, alternately,

$$
\begin{aligned}
C_{f}= & \left(\frac{64}{\mathrm{Re}}\right)+\frac{1}{\left(\frac{1}{2} \rho V^{2}\right)(L / D)} \\
& \times\left(\frac{4 \sigma\left(\cos \theta_{R}-\cos \theta_{s}+2\left(1+\cos \theta_{s}\right) C a^{0.5}\right)}{D}\right) .
\end{aligned}
$$

Further, the model is substantially simplified by narrowing the discussion only to completely wetting systems, due to the inherent lack of any model available for variation of the receding contact angle with $\mathrm{Ca}$. This means that the static contact angle and the receding contact angle will both be forced to zero (complete wetting), and Eq. (15) will become

$$
\begin{aligned}
C_{f} & =\frac{\Delta p_{\text {drive }}}{\left(\frac{1}{2} \rho V^{2}\right)(L / D)}=\frac{p_{1}-p_{2}}{\left(\frac{1}{2} \rho V^{2}\right)(L / D)} \\
& =\left(\frac{64}{\operatorname{Re}}\right)+\frac{16}{\left(\frac{1}{2} \rho V^{2}\right)(L / D)}\left(\frac{\sigma}{D}\right) C a^{0.5}
\end{aligned}
$$

Hence, the second term in Eq. (16) is supposed to account for the additional pressure drop due to the surface forces, which are over and above the classical HagenPoiseuille term. However, even this model severely underpredicts the experimental observation of Srinivasan et al [75], for water plug moving inside glass tube (partial wetting case), but may serve to a reasonably good measure for predicting the pressure drop of wetting fluids (such as methanol on glass), where $\mathrm{CAH}$ is low. The failure to predict for partially wetting (see figure 9b) fluid is attributed to the fact that the model calculates the pressure drop based on fully wetting assumption, where the hysteresis effects can be neglected [75]. However, based on the actual experimental advancing and receding contact angles reported by Srinivasan et al [75], it can be seen that Eq. (12) in itself is well able to match the experimentally measured pressure drop, since it takes into account CAH of partially wetting liquids. Thus, it follows that for partially non-wetting fluids, $\mathrm{CAH}$ will play a dominant role in the momentum transport of plugs moving inside capillary tubes or confined spaces. Such an observation was also made by Lips and Bonjour [81], even with a significantly wetting fluid (pentane on glass).

From the earlier discussions, it is clear that unless dynamic contact angle and the CL physics are included, hydrodynamics alone cannot predict the pressure drop correctly in such cases. It therefore becomes necessary to use a comprehensive model that accounts for the capillary pressure drop by incorporating the dynamic contact angles, so that Eq. (12) can be used to predict the total pressure drop required to move a plug of a partially wetting fluid inside a capillary tube. For such fluids, the relation between the dynamic and static contact angle (based on discussion in section 3.3), with the logarithmic correction of length scale is given by the Cox-Voinov model [45]:

$$
\theta_{d}=\theta_{s}+9 C a \ln \left(\frac{\beta l_{\text {macro }}}{l_{\text {micro }}}\right) \text {. }
$$

Using such an approach to model dynamic contact angle provides a holistic understanding of the flow physics as it explains CL motion and provides the apparent dynamic contact angle based on the CL velocity and the two length scales involved. While such an approach is highly successful in case of single-meniscus motion on an infinite flat surface (advancing interfaces), its applicability and extension to confined liquid plugs at low Bond numbers and specifically to dynamic receding contact angles needs further scrutiny. In order to match the experimentally observed 
pressure-drop data, we now need to model the pressure drop contribution due to surface effects, whose generic representation is given by the second term of Eq. (12). This requires the dynamic advancing and receding contact angles, which can be modelled based on the Cox-Voinov formulation of Eq. (7) (section 3.3). The second term in Eq. (7) provides the necessary correction in the static contact angles that the liquid plug makes inside the capillary tube during its motion. As $C a$ tends to zero, these corrections and therefore the net hysteresis $\left(\theta_{A}-\theta_{R}\right)$ vanish. Thus, once the static contact angle and Capillary number are known, the advancing and receding contact angles can be computed using Eq. (7), to find out the DCAH and the corresponding capillary pressure drop given by Eq. (12) (second term). It can be seen that in Eq. 7b, for small values of $\theta_{s}$, the receding angle may become negative. To avoid this situation, $\theta_{R}$ is assumed to be 0 for small values of $\theta_{s}$ (so as to avoid negative values for $\theta_{R}$ ), as has also been done by $\mathrm{Qu}$ et al [82].

Estimation of DCAH can also be done by alternate approaches, wherein the dependence of the advancing and receding contact angles and hence the DCAH as a function of $\mathrm{Ca}$ is given as [83] (Rodríguez-Valverde et al [84]) follows:

$$
\begin{gathered}
\theta_{A}=\theta_{s}+f H \\
\theta_{R}=\theta_{s}-(1-f) H
\end{gathered}
$$

where $H$ is the hysteresis $\left(\theta_{A}-\theta_{R}\right)$ itself and $f(0<f<1)$ is an asymmetry parameter, which relates to the resistance offered by the advancing and receding angles. If $f$ is low, then the deformation of advancing angle will be lower, hence offering lower resistance than the receding angle [84]. Qu et al [82] have used the above formulation to predict the capillary pressure drop component, i.e., the second term of Eq. (12). Figure 10 compares the prediction of DCAH as a function of $\mathrm{Ca}$, obtained from Eqs. (7) and (18); the relevant parameters for obtaining these curves are noted therein. It can be seen that, for small static contact angles $\left(\theta_{s}\right)$, since $\theta_{R}$ is assumed to be 0 in Eq. (7b) and (18b), the $\mathrm{CAH}$ increases, till a point where $\theta_{R}>0$, and then the hysteresis starts decreasing. Thus, as per these two models, for a given $\mathrm{Ca}$, there exists a unique static contact angle at which the dynamic hysteresis is a maximum and then it starts to decrease thereafter. It can also be seen that higher the $C a$, higher the hysteresis, and hence, as discussed earlier, higher the net pressure drop.

While the above two models, which are proposed for partially wetting fluids, show qualitatively similar trends as seen in figure 10, yet again, both the models are not able to predict the experimentally observed $\mathrm{CAH}$ for any of the fluids considered by Srinivasan et al [75]. Firstly, for low $\mathrm{Ca}\left(10^{-06}<\mathrm{Ca}<10^{-03}\right)$, both Eqs. (7) and (18) suggest that the resulting $\mathrm{CAH}$ (and hence the capillary pressure drop) is very low, which is not observed experimentally (see figures 3 and 10). Secondly, it is clearly seen from the experiments that D.I. water, having the maximum static contact angle, exhibits the highest $\mathrm{CAH}$ and capillary pressure drop, as compared with the surfactant solution or methanol. This behaviour is not even qualitatively suggested by any of the two models. Therefore, the results seem to suggest that a more comprehensive understanding (a)

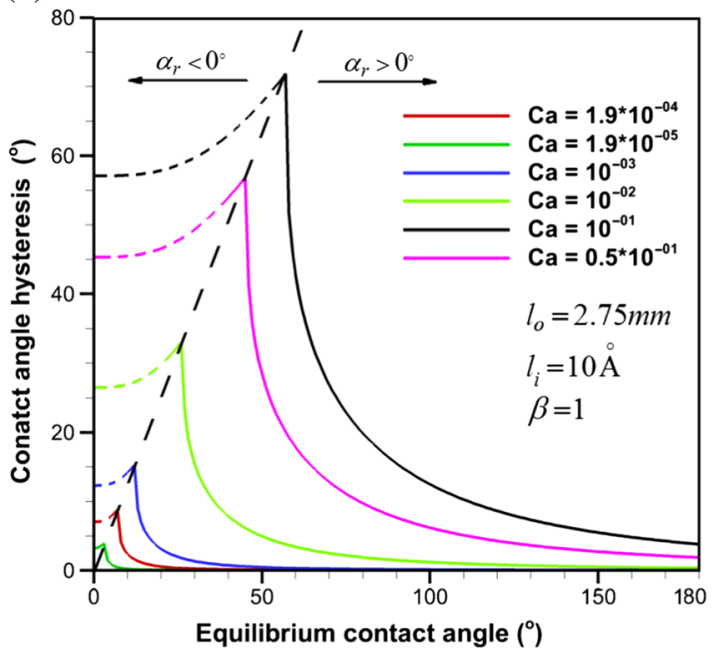

(b)

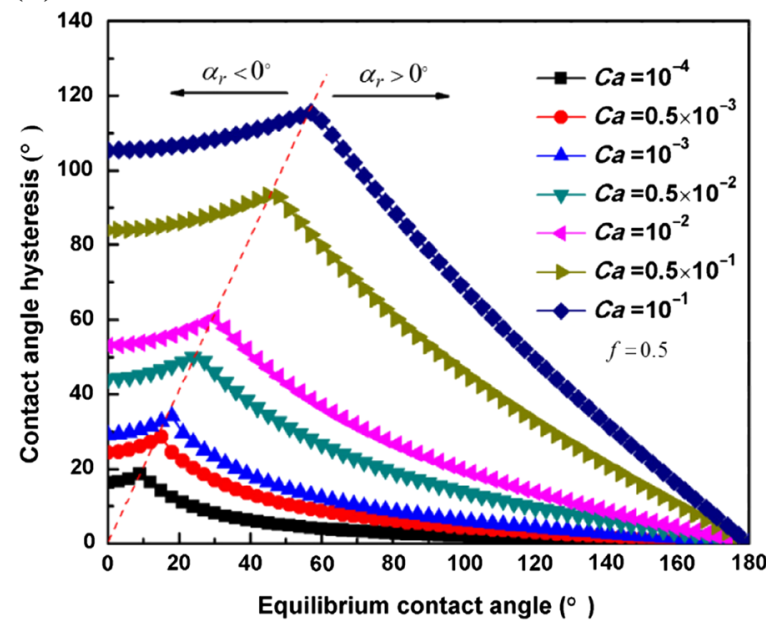

Figure 10. (a) Variation of contact angle hysteresis with static contact angles for different Capillary numbers based on the proposed model of Cox-Voinov, by including capillary length scale and molecular length scale to account for contact line motion. (b) Figure as adapted from Qu et al [82], where the dynamic contact angles are modeled as a non-linear function of contact angle hysteresis itself. Both the model has physically unrealistic prediction of receding contact angle for small static contact angles (i.e. for small static contact angles, receding contact angle becomes negative). This is overcome by assuming receding contact angle to be 0 for small values of static contact angle. 
(a)

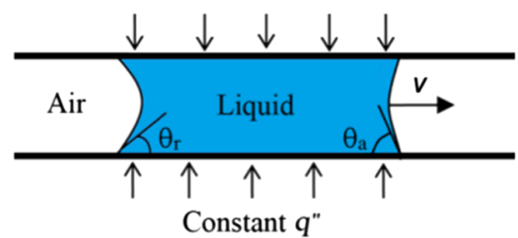

(b)

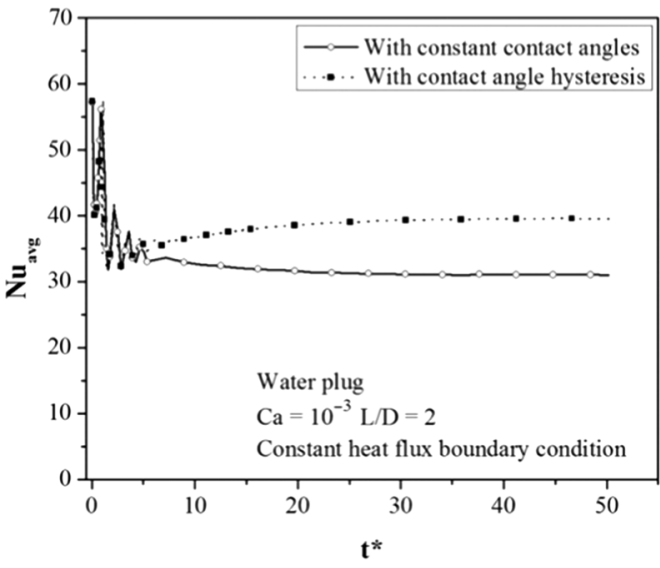

(c)
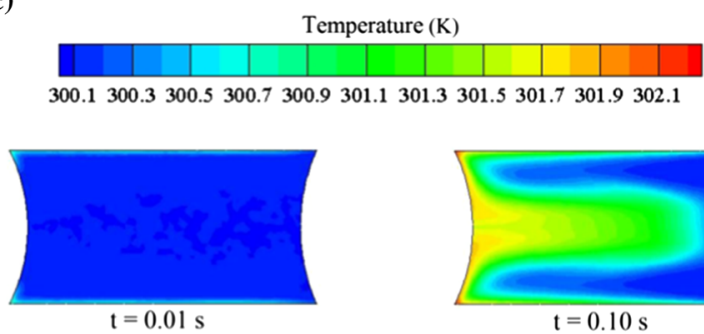

$\mathrm{t}^{*}=0.0717$

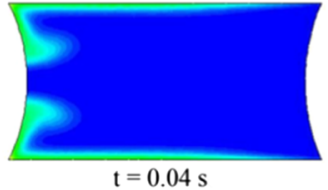

$t^{*}=0.2868$

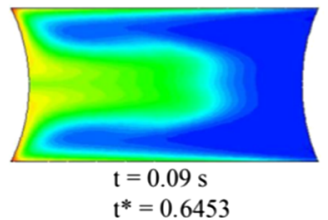

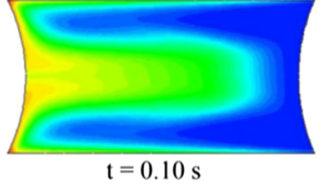

$t^{*}=0.7170$

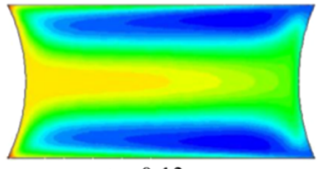

$\mathrm{t}=0.13 \mathrm{~s}$

$\mathrm{t}^{*}=0.9321$

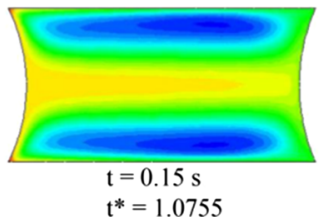

Figure 11. (a) Schematic of partially wetting isolated liquid plug moving at a steady velocity and subjected to constant wall heat flux. (b) Simulation result of temporal variation of average Nusselt number for water plug of $L / D=2$, moving at $\mathrm{Ca}=10 \times 10^{-03}$. It is clearly seen that the heat transfer is enhanced in the case of including contact angle hysteresis effect. (c) Thermal profile inside the liquid plug at different time instant.

of the CAH phenomena for partially wetting fluids when placed inside small diameter capillary tubes (with two distinct menisci) is required. The model for dynamic hysteresis will be highly beneficial for predicting the steadystate pressure drop during motion of partially wetting liquids [85].

4.2b Thermal behaviour of isolated liquid plug: The circulation patterns behind the moving meniscus, as seen from micro-PIV studies (section 4.1), enhance the local transport phenomena, as reported in available literature [15, 86-88, 91]. However, few studies have concentrated on explicitly showing the effect of $\mathrm{CAH}$ on heat transfer augmentation. Simulation studies [22, 89] have reported heat transfer enhancement for plug flows in mini/microchannel and have based it on the internal fluid circulation and thin film evaporation, but they have neglected the effect of CAH. As seen from sections 3.2 and 3.3, it is quite clear that the meniscus deformation during liquid plug motion, in the form ofCAH, contributes to substantially higher pressure drops. Hence, its effect on local heat transfer needs careful scrutiny. This is reported by Bajpai and Khandekar [90], wherein simulation of an isolated liquid plug moving inside a capillary tube was studied at low Capillary number, taking into account the DCAH.

Under steady-state motion of liquid plug, it is well established now, that the 'advancing' and 'receding' contact angles remain constant. Also, Hoffman [37] and Tanner
[36] suggested for low Capillary numbers that the advancing and receding contact angles are related to static contact angle and the Capillary number as [59] follows:

$$
\begin{gathered}
\theta_{A}=\theta_{s}+\frac{A|C a|}{3 \theta_{s}^{2}} \\
\theta_{R}=\theta_{s}-\frac{A|C a|}{3 \theta_{s}^{2}} .
\end{gathered}
$$

Using this linearized relation for $\mathrm{CAH}$ for a dry liquid plug motion at steady state, sensible heat transfer characteristic at different thermal boundary conditions (constant heat flux and constant wall temperature) was studied. Simulation result for different Capillary numbers (ranging from $10^{-05}$ to $10^{-03}$ ) and $L / D$ ratios $(1,2$ and 4 ) revealed that the inclusion of $\mathrm{CAH}$ leads to an enhanced local and average Nusselt number. This is attributed to the fact that the meniscus deformation changes the circulation pattern observed in the bulk liquid. Figure 11a shows the temporal variation of average Nusselt number for water at low Capillary number. The initial oscillation observed in the variation of average Nusselt number is attributed to the rapid motion of cold fluid in the central core of the liquid plug, which comes in contact with the tube wall because of higher frequency of internal circulation in water plug (because of lower viscosity). This is clearly demonstrated by the temperature contour of water plug at different time 
(a)

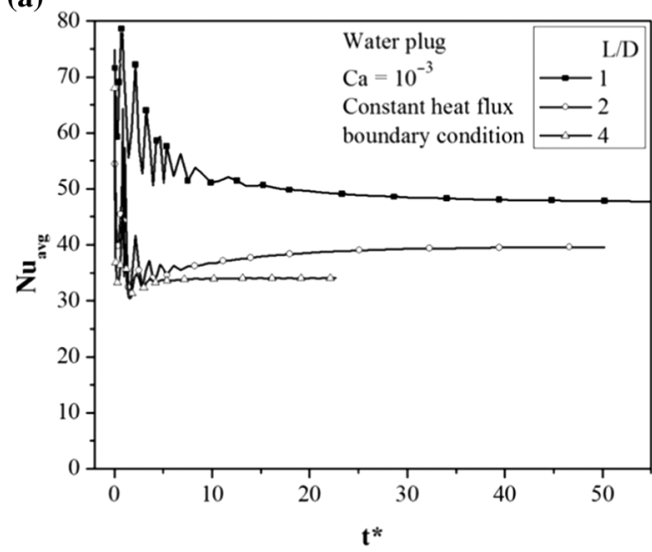

(b)

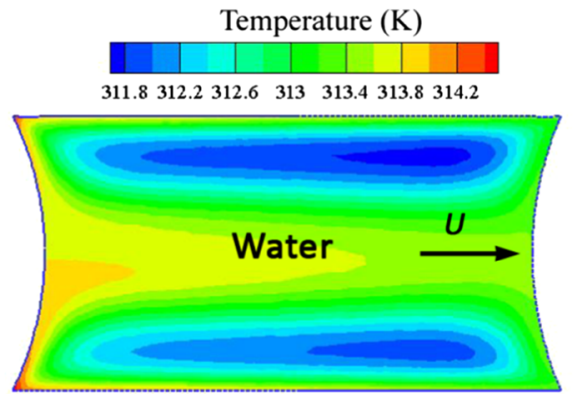

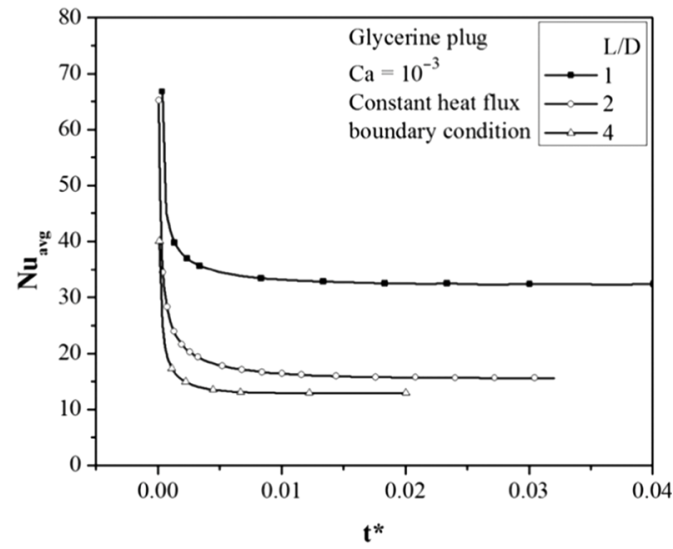

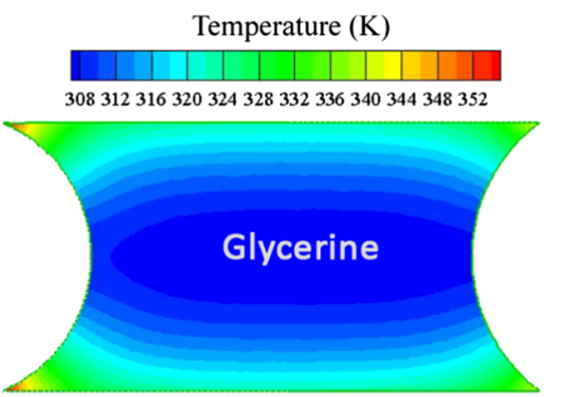

Figure 12. (a) Temporal variation of average Nusselt number under constant heat flux boundary condition for water plug and glycerine plug at same Capillary number $\left(10^{-03}\right)$ but different $L / D$. It can be seen that increasing the length deteriorate heat transfer. (b) Steady state temperature contours for Water ( $\mathrm{Pr}=7$, convection dominated) and glycerine ( $\mathrm{Pr}=6780$, diffusion dominated), corresponding to $\mathrm{Ca}=10^{-03}$ and $L / D=2$, for constant wall heat flux.

intervals as shown in figure $11 \mathrm{~b}$. As the internal flow circulation distributes the heat throughout the liquid plug, the Nusselt number decreases before reaching a constant value.

The study reveals the significance of $\mathrm{CAH}$ in determining the internal liquid circulation pattern, which enhances the local and average heat transfer. The work also highlights the effect of Prandtl number and $L / D$ ratio on local sensible thermal transport. For higher Prandtl number fluids, the dominant mode of heat transfer is through diffusion and the circulation (and hence the meniscus shape) has little effect on heat transfer enhancement as compared with that of lower Prandtl number fluids [89]. This is depicted in figure 12, where it is seen that increasing Prandtl number does not favour thermal transport via circulating fluid. Finally, the $L / D$ ratio of liquid plug also plays a dominant role in thermal transport. Figure 12a shows that increasing the length of liquid plug deteriorates thermal performance as increasing the length increases the time scale for one complete circulation inside the bulk liquid, thereby decreasing the circulation effect. It is also seen from the average Nusselt number that the steady-state Nusselt number for water is greater than that of glycerine for the same $L / D$. This is attributed to the higher Prandtl number of glycerine plug, which renders diffusion-dominant thermal transport.

\section{Future scope and challenges}

Experimental data on the explicit effect of DCAH on thermal transport of partially wetting fluids in confined geometries is rather limited. Accordingly, thermal transport models for such systems are also not readily available in open literature. The difficulty of measuring local temperature profile stems from the miniature geometry, where intrusive methods fail. Infra-red thermograph (IRT) is extensively used to study temperature profile of the surface in case of Taylor flows [87, 91]; however, obtaining temperature profile within the liquid plug still remains challenging. Optical techniques such as Laser-Induced Fluorescence Thermograph (LIFT), etc., are attracting focus to overcome these difficulties and shed light on thermal transport in such partially wetting liquid plugs.

\section{Summary and conclusions}

The review attempts to bring out the transport phenomena of a unit cell of Taylor liquid plugs. The PIV visualization study of a single meniscus shows the origin of circulation pattern because of the meniscus and also brings out the effect of wettability on circulation pattern. For single-meniscus motion 
inside a mini/micro-channel, it was seen that the hydrodynamic friction factor increased by increasing wettability.

An experimental study to understand the 'dry' plug motion inside capillary tubes reveals that the net friction is nearly independent of the length of the plug as the hydrodynamic component is heavily overshadowed by the additional dissipation due to capillary forces due to dynamic contact angle hysteresis and surface forces at the threephase contact line. It is thus conclusive that the prediction of flow friction factor using the classical Hagen-Poiseuille relation is not valid for such flow conditions. The applicability of Cox-Voinov model to motion of an isolated liquid plug is also seen to lead to erroneous results for partially wetting fluids. Considering only the hydrodynamic component of friction, the available models predict a lower pressure drop for partially wetting fluids, which is not observed in experiments due to surface effects coming into play. It is therefore necessary to model the pressure drop of partially wetting liquid slugs comprehensively taking into account contact angle hysteresis and contact line motion. Modelling this pressure drop is of prime importance to set the operating conditions of engineering devices such as labon-chips, two-phase catalytic reactors, pulsating heat pipes, etc. Further, liquid plug motion at high Capillary number needs to be studied to understand the effect of inertia.

Sensible heat transfer augmentation associated with isolated dry plug motion was also studied and reported. It is found that the heat transfer is significantly higher than that of the corresponding single-phase flow, which is attributed to the internal circulation. These circulations are also affected by contact angle hysteresis. The heat transfer of such isolated liquid plug flow is also a strong function of length of the liquid plug, the Prandtl number of the liquid and the Capillary number. Thus, a practical model for transport coefficients of Taylor flows needs to incorporate all the effects that were discussed. Also, such local study of a unit cell of Taylor flows will uncover more insight into the physics of such flows, which can be used to develop a global model, for designing devices for practical applications.

\section{Acknowledgements}

This research work was done as part of the activities under the sponsored project from the Indo-French Center for Promotion of Advanced Research (IFCPAR/CEFIPRA), New Delhi, under Grant Number 4408-1. The authors are grateful for useful discussion with project partners Prof. J. Bonjour and Prof. F. Lefevre, both from Centre for Thermal Sciences (CETHIL), INSA-Lyon, France.

\section{Nomenclature}

$$
\begin{array}{ll}
\text { A } & \text { Hamaker constant }(\mathrm{J}) \\
\text { Bo } & \text { Bond number }\left(\frac{\rho g D^{2}}{\sigma}\right)
\end{array}
$$

\author{
$\mathrm{Ca}$ Capillary number $\left(\frac{\mu V}{\sigma}\right)$ \\ $C_{f} \quad$ Friction factor (-) \\ $D \quad$ Diameter (m) \\ $e \quad$ Liquid film thickness (m) \\ $g \quad$ Acceleration due to gravity $\left(\mathrm{m} / \mathrm{s}^{2}\right)$ \\ $h \quad$ Liquid thin-film thickness (m) \\ $l, L \quad$ Length scale, length (m) \\ $P \quad$ Pressure drop $\left(\mathrm{N} / \mathrm{m}^{2}\right)$ \\ Pr Prandtl number $\left(\frac{v}{\alpha}\right)$ \\ $r \quad$ Radius (m) \\ Re Reynolds number $\left(\frac{\rho V D}{\mu}\right)$ \\ $S \quad$ Spreading coefficient $(\mathrm{N} / \mathrm{m})$ \\ $V \quad$ Velocity $(\mathrm{m} / \mathrm{s})$ \\ We Weber number $\left(\frac{\rho D V^{2}}{\sigma}\right)$
}

\section{Greek symbols}

$\alpha \quad$ Thermal diffusivity $\left(\mathrm{m}^{2} / \mathrm{s}\right)$

$\theta \quad$ Contact angle $\left({ }^{\circ}\right)$

$\mu \quad$ Dynamic viscosity ( $\mathrm{Pa} \mathrm{s}$ )

$v \quad$ Kinematic viscosity $\left(\mathrm{m}^{2} / \mathrm{s}\right)$

$\rho$ Density $\left(\mathrm{kg} / \mathrm{m}^{3}\right)$

$\Pi$ Disjoining pressure $\left(\mathrm{N} / \mathrm{m}^{2}\right)$

$\sigma$ Surface tension $(\mathrm{N} / \mathrm{m})$

$\tau$ Shear stress $\left(\mathrm{N} / \mathrm{m}^{2}\right)$

\section{Subscripts}

A Advancing

c Characteristics, critical, capillary

d Dynamic

$v$ Vapour/gas phase

$R \quad$ Receding

$s \quad$ Static, solid phase

$l \quad$ Liquid phase

$w \quad$ Wall

\section{References}

[1] Devesenathipathy S, Santiago J G, Werely S T, Meinhart C D and Takhera K 2003 Particle imaging techniques for microfabricated fluidic systems. Exp. Fluids 34: 504-514

[2] Ghiaasiaan S M and Abdel-Khalik S I 2001 Two-phase flow in microchannels. Adv. Heat Transf. 34: 145-254

[3] Spernjak D, Prasad A K and Advani S G 2007 Experimental investigation of liquid water formation and transport in a transparent single-serpentine PEM fuel cell. J. Power Sources 170: 334-344 
[4] Steijn V, van Kreutzer M T and Kleijn C R 2008 Velocity fluctuations of segmented flows in microchannels. Chem. Eng. J. 135: 159-165

[5] Triplett K A, Ghiaasiaan S M, Khalik A, Le-Mouel S I A and McCord B N 1999 Gas-liquid two-phase flow in microchannels. Part I: two-phase flow pattern. Int. J. Multiphase Flow 25: 377-394

[6] Triplett K A, Ghiaasiaan S M, Khalik A, Le-Mouel S I A and McCord B N 1999 Gas-liquid two-phase flow in microchannels. Part II: void fraction and and pressure drop. Int. J. Multiphase Flow 25: 395-410

[7] Brauner N and Moalem M D 1992 Identification of the range of small diameter conduits, regarding two-phase flow pattern transitions. Int. Commun. Heat Mass Transf. 19(1): 29-39

[8] Kandlikar S G 2002 Fundamental issues related to flow boiling in mini-channels and micro-channels. Exp. Therm. Fluid Sci. 26(2): 389-407

[9] Khandekar S, Panigrahi P K, Lefevre F and Bonjour J 2010 Local hydrodynamics of flow in a pulsating heat pipe: a review. Front. Heat Pipes 1: 023003

[10] Bretherton F P 1961 The motion of long bubbles in tubes. J. Fluid Mech. 10: 166-188

[11] Thome J R, Dupont V and Jacobi A M 2004 Heat transfer model for evaporation in microchannels. Part I: presentation of the model. Int. J. Heat Mass Transf. 47: 3375-3385

[12] Fairbrother F and Stubbs A E 1935 The bubble-tube method of measurement. J. Chem. Soc. 1: 527-529

[13] Taylor G I 1961 Deposition of viscous fluid on the wall of a tube. J. Fluid Mech. 10: 161-165

[14] Angeli P and Gavriilidis S M 2006 Hydrodynamics of Taylor flow in small channels: a review. Proc. IMechE Part C: J. Mech. Eng. Sci. 222: 2367-2371

[15] Thulasidas T C, Abraham M A and Cerro R L 1997 Flow patterns in liquid slugs during bubble train flow inside capillaries. Chem. Eng. Sci. 52(17): 2947-2962

[16] Cubaud T and Ho C M 2004 Transport of bubbles in square microchannels. Phys. Fluids 16(12): 4575-4585

[17] Serizawa A, Feng Z and Kawara Z 2002 Two-phase flow in microchannels. Exp. Therm. Fluid Sci. 26: 703-714

[18] Kreutzer M T, van der Eijnden M G, Kapteijn F, Moulijn J A and Heiszwolf J J 2005 The pressure drop experiments to determine slug lengths in multiphase monoliths. Catal. Today 105: 667-672

[19] Warnier M J F, de Croon M H J M, Rebrov E V and Schouten J C 2010 Pressure drop of gas-liquid Taylor flow in round micro-capillaries for low to intermediate Reynolds numbers. Microfluid. Nanofluid. 8: 33-45

[20] Walsh E, Muzychka Y, Walsh P, Egan V and Punch J 2009 Pressure drop in two phase slug/bubble flows in mini scale capillaries. Int. J. Multiphase Flow 35: 879-884

[21] Abadie T, Aubin J, Legendre D and Xuereb C 2012 Hydrodynamics of gas-liquid Taylor flow in rectangular channel. Microfluid. Nanofluid. 12: 355-369

[22] He Q, Hasegawa Y and Kasagi N 2010 Heat transfer modelling of gas-liquid slug flow without phase change in a micro tube. Int. J. Heat Fluid Flow 31: 126-136

[23] Zhao T S and Bi Q C 2001 Co-current air water two phase flow patterns in vertical triangular micro channels. Int. J. Multiphase Flow 27(5): 765-782

[24] de Gennes P G 1985 Wetting: statics and dynamics. Rev. Mod. Phys. 57: 827-863
[25] Kirkwood J and Buff F 1949 The statistical mechanical theory of surface tension. J. Chem. Phys. 17: 338-343

[26] Carey V P 2007 Liquid-vapor phase-change phenomena. New York, USA: Taylor \& Francis

[27] Bonn D, Eggers J, Indekeu J, Meunier J and Rolley E 2009 Wetting and spreading. Rev. Mod. Phys. 81: 739-805

[28] Kwok D Y and Neumann A W 1999 Contact angle measurement and contact angle interpretation. Adv. Colloid Interface Sci. 81(3): 167-249

[29] Decker E L, Frank B, Suo Y and Garoff S 1999 Physics of contact angle measurement. Colloids Surf. A Physicochem. Eng. Aspects 156: 177-189

[30] Huh C and Scriven L E 1971 Hydrodynamic model of steady movement of a solid/liquid/fluid contact line. J. Colloid Interface Sci. 35: 85-101

[31] Blake T D 2006 The physics of moving wetting lines. $J$. Colloid Interface Sci. 299(1): 1-13

[32] Eggers J 2005 Contact line motion for partially wetting fluids. Phys. Rev. E 72: 061605

[33] Eggers J 2005 Existence of receding and advancing contact lines. Phys. Fluids 17: 082106

[34] Rose W and Heins R W 1962 Moving interfaces and contact angle rate-dependency. J. Colloid Sci. 17: 39-48

[35] Dussan V E B and Davis S H 1974 On the motion of a fluidfluid interface along a solid surface. J. Fluid Mech. 65: 71-95

[36] Tanner L H 1979 The spreading of silicone oil drops on horizontal surfaces. J. Phys. D Appl. Phys. 12: 1473-1484

[37] Hoffman R L 1975 A study of advancing interface. J. Colloid Interface Sci. 50: 228-241

[38] Huh C and Mason S G 1977 The steady movement of a liquid meniscus in a capillary tube. J. Fluid Mech. 81(3): 401-419

[39] Marmur A 1994 Contact angle hysteresis on heterogeneous smooth surfaces. J. Colloid Interface Sci. 168: 40-46

[40] Tadmor R 2011 Approaches in wetting phenomena. Soft Matter 75(5): 1577-1580

[41] Morris S J S 2003 The evaporating meniscus in a channel. J. Fluid Mech. 494: 297-317

[42] Potash M and Wayner P C 1972 Evaporation from a twodimensional extended meniscus. Int. J. Heat Mass Transf. 15: $1851-1863$

[43] Hardy W P 1919 The spreading of fluids on glass. Philos. Mag. 38: 49

[44] Kavehpour H P, Ovryn B and McKinley G H 2003 Microscopic and macroscopic structure of the precursor layer in spreading viscous drops. Phys. Rev. Lett. 91: 196104

[45] de Gennes P G, Hua X and Levinson P 1990 Dynamics of wetting: local contact angles. J. Fluid Mech. 212: 55-63

[46] Snoeijer J H and Anderotti B 2008 A microscopic view on contact angle selection. Phys. Fluids 20: 057101

[47] Derjaguin B V, Churaev N V and Muller V M 1987 Surface forces. New York, USA: Plenum Press

[48] Israelachvili J N 2009 Intermolecular and surface forces, 3rd ed. London: Academic Press

[49] Derjaguin B V and Churaev N V 1974 Structural component of disjoining pressure. J. Colloid Interface Sci. 49(2): 249-255

[50] Popescu M N, Oshanin G, Dietrich S and Cazabat A M 2012 Precursor films in wetting phenomena. J. Phys. Condens. Matter 24: 243102 (30pp) 
[51] Cazabat A M 1987 How does a droplet spread? Contemp. Phys. 28(4): 347-364

[52] Dussan V E B 1979 On the spreading of liquids on solid surfaces: static and dynamic contact lines. Annu. Rev. Fluid Mech. 11: 371-400

[53] Voinov O V 1976 Hydrodynamics of wetting. Fluid Dyn. 11: 714-721

[54] Cox R G 1986 The dynamics of the spreading of liquids on a solid surface. Part 1. Viscous flow. J. Fluid Mech. 168: 169-194

[55] Diez J A, Kondic L and Bertozzi A 2000 Global models for moving contact lines. Phys. Rev. E 63: 1-13

[56] Snoeijer J H and Andreotti B 2013 Moving contact lines: scales, regimes and dynamical transitions. Annu. Rev. Fluid Mech. 45: 269-292

[57] Gao L and McCarthy T J 2006 Contact angle hysteresis explained. Langmuir 22: 6234-6237

[58] Eral H B, 't Mannetje D J C M and Oh J M 2013 Contact angle hysteresis: a review of fundamentals and applications. Colloid Polym. Sci. 291: 241-260

[59] Berthier J 2008 Microdrops and digital microfluidics. New York, USA: William Andrew Inc

[60] Joanny J F and Robbins O M 1990 Motion of a contact line on a heterogeneous surface. J. Chem. Phys. 92: 3206-3212

[61] Tanguy A and Vettorel T 2004 From weak to strong pinning i: a finite size study. Eur. Phys. J. B 38: 71-82

[62] Aussillous P and Quéré D 2000 Quick deposition of a fluid on the wall of a tube. Phys. Fluids 12: 2367-2371

[63] Han Y and Shikazono N 2009 Measurement of the liquid film thickness in micro tube slug flow. Int. J. Heat Fluid Flow 30(5): 842-853

[64] Quéré D 1991 On minimal velocity of forced spreading in partial wetting. C. R. Acad. Sci. 313: 313-318

[65] Redon C, Brochard-Wyart F and Rondelez F 1991 Dynamics of dewetting. Phys. Rev. Lett. 66: 715-718

[66] Thille U 2003 Open questions and promising new fields in dewetting. Eur. Phys. J. E 12: 409-416

[67] Flitton J C and King J R 2004 Surface tension driven dewetting of Newtonian and power law fluids. J. Eng. Math. 50: 241-266

[68] Snoeijer J H and Eggers J 2010 Asymptotics of the dewetting rim. Phys. Rev. E 82: 056314

[69] Egorov A G, Kornev K G and Neimark A V 2003 Meniscus motion in a pre-wetted capillary. Phys. Fluids 15(10): 3134-3143

[70] Waters S L and Grotberg J B 2002 The propagation of a surfactant laden liquid plug in a capillary tube. Phys. Fluids 14(2): 471-480

[71] Rao M, Lefèvre F, Khandekar S and Bonjour J 2013 Understanding transport mechanism of a self-sustained thermally driven oscillating two-phase system in a capillary tube. Int. J. Heat Mass Transf. 65: 451-459

[72] Safavieh R and Juncker D 2013 Capillarics: pre-programmed, self-powered microfluidic circuits built from capillary elements. Lab Chip 13(21): 4180-4189

[73] Rapolu P and Son S Y 2011 Characterization of wettability effects on pressure drop of two-phase flow in microchannel. Exp. Fluids 51(4): 1101-1108
[74] Rana G R, Sikarwar B S, Khandekar S and Panigrahi P K 2014 Hydrodynamics of a confined meniscus in a square capillary tube at low capillary numbers. Front. Heat Pipes 5: 1

[75] Srinivasan V, Bouamrane N, Khandekar S, Lefevre F and Bounjour J 2015 Motion of an isolated liquid plug inside a capillary tube: effect of contact angle hysteresis. Exp. Fluids 56: 14

[76] Bico J and Quéré D 2001 Falling slugs. J. Colloid Interface Sci. 243(1): 262-264

[77] Jensen O E 2000 Draining collars and lenses in liquid-lined vertical tubes. J. Colloid Interface Sci. 221: 38-48

[78] Lunati I and Or D 2009 Gravity-driven slug motion in capillary tubes. Phys. Fluids 21: 052003

[79] Ma Y D 2012 Motion effect on the dynamic contact angles in a capillary tube. Microfluid. Nanofluid. 12: 671-675

[80] Bracke M, De Voeght F and Joos P 1989 The kinetics of wetting: the dynamic contact angle. Prog. Colloid Polym. Sci. 9: 142-149

[81] Lips S and Bonjour J 2007 Oscillating two-phase flow in a capillary tube: experiments and modeling. In: Proceedings of the 14th International Heat Pipe Conference, Florianopolis, Brazil

[82] Qu J, Wang Q, Li C, Han X and He Z 2014 A simple model for Taylor flow induced contact angle hysteresis and capillary pressure inside mini/micro-scale capillary tubes. Int. J. Heat Mass Transf. 78: 1004-1007

[83] Neumann A W and Spelt J K 1996 Applied surface thermodynamics. New York, USA: Marcel Dekker Inc

[84] Rodríguez-Valverde M A, Ruiz-Cabello F J, Gea-Jódar P M, Kamusewitz H, Cabrerizo-Vílchez M A 2010 A new model to estimate the Young contact angle from contact angle hysteresis measurements. Colloids Surf. A. 365:21-27

[85] Kuchin I V and Starov V M 2016 Hysteresis of the contact angle of a meniscus inside a capillary with smooth, homogenous solid walls. Langmuir 32(21): 5333-5340

[86] King C, Walsh E and Grimes R 2007 PIV measurements of flow within plugs in a microchannel. Microfluid. Nanofluid. 3: 463-472

[87] Mehta B and Khandekar S 2014 Measurement of local heat transfer coefficient during gas-liquid Taylor bubble train flow by infrared thermography. Int. J. Heat Fluid Flow 45: $41-52$

[88] Son S Y and Allen J S 2004 Visualization of wettability effects on micro-channel two-phase flow resistance. J. Heat Transf. 126: 498-499

[89] Talimi V, Muzychka Y S and Kocabiyik S 2012 Numerical simulation of pressure drop and heat transfer of two-phase slug flows in microtubes using moving frame of reference technique. Int. J. Heat Mass Transf. 55: 6463-6472

[90] Bajpai A K and Khandekar S 2012 Thermal transport behavior of a liquid plug moving inside a dry capillary tube. Heat Pipe Sci. Technol. Int. J. 3(2-4): 97-124

[91] Mehta B and Khandekar S 2014 Taylor bubble-train flow and heat transfer in the context of pulsating heat pipes. Int. J. Heat Mass Transf. 79: 279-290 